\title{
Supersymmetric QCD corrections to single top quark production at hadron colliders
}

\author{
Jia Jun Zhang, Chong Sheng Li, Zhao Li, and Li Lin Yang \\ Department of Physics, Peking University, Beijing 100871, China
}

(Dated: June 24, 2018)

\begin{abstract}
We present the calculations of the supersymmetric QCD corrections to the total cross sections for single top production at the Fermilab Tevatron and the CERN Large Hadron Collider in the minimal supersymmetric standard model. Our results show that for the s-channel and t-channel, the supersymmetric QCD corrections are at most about $1 \%$, but for the associated production process $p p \rightarrow t W$, the supersymmetric QCD corrections increase the total cross sections significantly, which can reach about $6 \%$ for most values of the parameters, and the supersymmetric QCD corrections should be taken into consideration in the future high precision experimental analysis for top physics.
\end{abstract}

PACS numbers: 12.38.Bx, 12.60.Jv, 14.65.Ha 


\section{INTRODUCTION}

The search for single top quark production is one of the major aims of both the Fermilab Tevatron and the CERN Large Hadron Collider(LHC) [1, 2], because it can probe the electroweak sector of the Standard Model(SM), in contrast with the dominant QCD pair production mechanism, and provide a consistency check on the measured parameters of the top quark in the QCD pair production process [3, 4, 5, 6]. Further more, the mass of top quark is comparable to the electroweak $(\mathrm{EW})$ symmetry breaking scale, and it can play a role of wonderful probe for the EW symmetry breaking mechanism and new physics, especially the minimal supersymmetry standard model(MSSM) as a very popular model beyond the SM, via non-standard couplings [4, 7, 8, 9, 10, 11, 12, 13], loop effects [14, 15, 16, 17, 18], etc., in the processes for single top quark production.

At the LHC single top quarks are produced primarily via the t-channel [19],

$$
q+b \rightarrow q^{\prime}+t
$$

the quark annihilation process(s-channel) [20, 21],

$$
q+\bar{q}^{\prime} \rightarrow t+\bar{b}
$$

and the associated production process [3, 22],

$$
g+b \rightarrow t+W^{-}
$$

which can reliably be predicted in the SM, and their leading order(LO) results are summarized in Table [2]. As a high-luminosity LHC would run soon and allow accurate measure-

TABLE I: The LO results for single top production at the LHC

\begin{tabular}{|c|c|c|c|}
\hline process: & t-channel & s-channel & $\mathrm{Wt}$ \\
\hline$\sigma(p b):$ & $156 \pm 8$ & $6.6 \pm 0.6$ & $14.0_{-2.8}^{+3.8}$ \\
\hline
\end{tabular}

ments of these cross sections with a statistical uncertainty of less than $2 \%$ [1, 22, 23, 24, 25], at this level of experimental accuracy, calculations of the radiative corrections are necessary to test the predictions of the SM and to investigate loop effects arising from new physics. The QCD corrections to the total cross sections of the three channels are about $-10 \%$, 
$+50 \%$ and $+50 \%$, respectively [2, 22, 26, 27, 28, 29]. For t-channel, the SM EW corrections are about $10 \%$ and the supersymmetric(SUSY) EW corrections are a few percent [30] at the LHC, for s-channel, the combined effects of SUSY QCD, SUSY EW and the Yukawa couplings can exceed $10 \%$ for small $\tan \beta(<2)$ but are only a few percent for $\tan \beta>2$ at the Tevatron [16, 17], and for the associated production at the LHC, the SM EW and the SUSY corrections have been calculated and analyzed in Ref.[31]. But at the LHC, the SUSY QCD corrections to the three channels have not been calculated yet. As the associated production process involves the QCD coupling in initial state, obviously, the SUSY QCD corrections are significant for this channel. In order to fill in the blanks in the relevant radiative corrections to the channels considered here, in this paper we present the calculations of the SUSY QCD corrections to the three channels at the CERN LHC, mainly concerning the associated production channel, and also give the updated numerical calculations to the s-channel process at the Tevatron[32].

Our paper is organized as follows. In Section II we will give the analytic results in terms of the well-known standard notation of one-loop Feynman integrals for the associated channel, s-channel and t-channel. In Section III we will present our numerical results with discussions of their implications.

\section{ANALYTIC RESULTS}

\section{A. Associated Production}

In our calculations we use dimensional reduction to control all the ultraviolet divergences in the virtual loop corrections. We adopt the on-mass-shell renormalization scheme [33] for the top quark mass and the wave functions renormalization, and set all the other quark masses as zero. The QCD coupling constant $g_{s}$ is renormalized in the modified minimal subtraction scheme $(\overline{\mathrm{MS}})$ except that the divergences associated with the top quark and colored SUSY particle loops are subtracted at zero momentum[34]. Denoting $\psi_{q 0}, m_{q 0}, g_{s 0}$ and $A_{\mu 0}$ as bare quark wave functions, quark masses, strong coupling constant and gluon wave function respectively, the relevant renormalization constants are then defined as

$$
\begin{aligned}
\psi_{q 0} & =\left(1+\delta Z_{L}^{q q}\right)^{1 / 2} \psi_{q L}+\left(1+\delta Z_{R}^{q q}\right)^{1 / 2} \psi_{q R}, \\
m_{q 0} & =m_{q}+\delta m_{q}
\end{aligned}
$$




$$
\begin{aligned}
A_{\mu 0} & =\left(1+\delta Z_{A A}\right)^{1 / 2} A_{\mu} \\
g_{s 0} & =\left(1+\delta Z_{g}\right) g_{s}
\end{aligned}
$$

After calculating the self-energy and vertex diagrams in Fig,1, we obtain the explicit expressions of all necessary renormalization constants as follows:

$$
\begin{aligned}
\delta Z_{g}= & -\frac{\alpha_{s}\left(\mu^{2}\right)}{4 \pi}\left\{\frac{\beta_{0}}{2}\left[\frac{1}{\epsilon}-\gamma_{E}+\ln (4 \pi)\right]+\frac{N}{3} \ln \left(\frac{M_{\tilde{g}}^{2}}{\mu^{2}}\right)+\sum_{u=u, c, t}^{i=1,2} \frac{1}{12} \ln \left(\frac{M_{\tilde{u}_{i}}^{2}}{\mu^{2}}\right)+\sum_{d=d, s, b}^{i=1,2} \frac{1}{12} \ln \left(\frac{M_{\tilde{d}_{i}}^{2}}{\mu^{2}}\right)\right\}, \\
\delta Z_{A A}= & -\frac{3 \alpha_{s}}{2 \pi} B_{1}\left(0, M_{\tilde{g}}^{2}, M_{\tilde{g}}^{2}\right)+\frac{3 \alpha_{s}}{\pi} \frac{\partial}{\partial p^{2}} B_{00}\left(0, M_{\tilde{g}}^{2}, M_{\tilde{g}}^{2}\right) \\
& -\frac{\alpha_{s}}{2 \pi} \sum_{u}^{3} \sum_{i}^{2} \frac{\partial}{\partial p^{2}} B_{00}\left(0, M_{\tilde{u}_{i}}^{2}, M_{\tilde{u}_{i}}^{2}\right)-\frac{\alpha_{s}}{2 \pi} \sum_{d}^{3} \sum_{i}^{2} \frac{\partial}{\partial p^{2}} B_{00}\left(0, M_{\tilde{d}_{i}}^{2}, M_{\tilde{d}_{i}}^{2}\right), \\
\delta m_{t}= & \frac{2 \alpha_{s} M_{\tilde{g}}}{3 \pi} \sum_{i}^{2} B_{0}\left(m_{t}^{2}, M_{\tilde{g}}^{2}, M_{\tilde{t}_{i}}^{2}\right) U_{i 1}^{t} U_{i 2}^{t *}+\frac{\alpha_{s} m_{t}}{3 \pi} \sum_{i}^{2} B_{1}\left(m_{t}^{2}, M_{\tilde{g}}^{2}, M_{\tilde{t}_{i}}^{2}\right)\left(U_{i 1}^{t} U_{i 1}^{t *}+U_{i 2}^{t} U_{i 2}^{t *}\right), \\
\delta Z_{(L, R)}^{t t}= & -\frac{2 \alpha_{s}}{3 \pi} \sum_{i}^{2} B_{1}\left(m_{t}^{2}, M_{\tilde{g}}^{2}, M_{\tilde{t}_{i}}^{2}\right) U_{i(1,2)}^{t} U_{i(1,2)}^{t *}-\frac{4 \alpha_{s} M_{\tilde{g}} m_{t}}{3 \pi} \sum_{i}^{2} \frac{\partial}{\partial p^{2}} B_{0}\left(m_{t}^{2}, M_{\tilde{g}}^{2}, M_{\tilde{t}_{i}}^{2}\right) U_{i 1}^{t} U_{i 2}^{t *} \\
& -\frac{2 \alpha_{s} m_{t}^{2}}{3 \pi} \sum_{i}^{2} \frac{\partial}{\partial p^{2}} B_{1}\left(m_{t}^{2}, M_{\tilde{g}}^{2}, M_{\tilde{t}_{i}}^{2}\right)\left(U_{i 1}^{t} U_{i 1}^{t *}+U_{i 2}^{t} U_{i 2}^{t *}\right), \\
\delta Z_{(L, R)}^{q q}= & -\frac{2 \alpha_{s}}{3 \pi} \sum_{i}^{2} B_{1}\left(0, M_{\tilde{g}}^{2}, M_{\tilde{q}_{i}}^{2}\right) U_{i(1,2)}^{q} U_{i(1,2)}^{q *} \quad q=u, d, s, c, b,
\end{aligned}
$$

where $\beta_{0}=\left[-\frac{2}{3}(N+1)-\frac{1}{3}\left(n_{f}+1\right)\right], B_{i j k \ldots}$ are the two-point functions, $U_{i j}^{q}$ are the mixing matrices of the squarks, $\gamma_{E}$ is the Euler constant, and $\mu$ is the renormalization scale.

Including the SUSY QCD corrections, the renormalized amplitudes can be written as

$$
M_{\text {ren }}^{A}=M_{0}^{A}+M_{v i r}^{A}+M_{\text {count }}^{A},
$$

where $M_{0}^{A}$ is the LO amplitude, $M_{v i r}^{A}$ represents the SUSY QCD corrected amplitude from the one-loop self-energy, vertex, and box diagrams, and $M_{\text {count }}^{A}$ is the corresponding counterterm for the self-energy corrections and vertex corrections, respectively.

The related Feynman diagrams which contribute to the LO amplitude $M_{0}^{A}$ are shown in Fig!2 (a) and (b). The LO amplitude $M_{0}^{A}$ is given by

$$
\begin{aligned}
M_{0}^{A} & =M_{s}^{A}+M_{t}^{A} \\
& =\frac{e g_{s} V_{t b}}{\sqrt{2} \sin \theta_{W}}\left[\frac{1}{s}\left(2 A_{10}-A_{7}-2 A_{8}\right)+\frac{1}{t-m_{t}^{2}}\left(2 A_{15}-2 A_{9}-A_{7}\right)\right],
\end{aligned}
$$


where $V_{i j}$ are the elements of CKM matrix, s, t, $\mathrm{u}$ are the Mandelstam invariants, which are defined as,

$$
\begin{aligned}
s & =\left(k_{1}+k_{2}\right)^{2}=\left(k_{3}+k_{4}\right)^{2}, \\
t & =\left(k_{1}-k_{3}\right)^{2}=\left(k_{2}-k_{4}\right)^{2}, \\
u & =\left(k_{1}-k_{4}\right)^{2}=\left(k_{2}-k_{3}\right)^{2},
\end{aligned}
$$

where $k_{1}$ and $k_{2}$ denote the momentum of the incoming particles, $k_{3}$ and $k_{4}$ the outgoing particles, while $A_{m}$ are the reduced standard matrix elements given by

$$
\begin{aligned}
& A_{1,22}=\left(\epsilon_{1}^{a} \cdot \epsilon_{4}^{*}\right) \bar{u}^{b}\left(k_{3}\right) P_{R, L} u^{c}\left(k_{2}\right)\left(T^{a}\right)_{b c}, \\
& A_{2,3}=\left(\epsilon_{1}^{a} \cdot k_{2}\right)\left(\epsilon_{4}^{*} \cdot k_{1,2}\right) \bar{u}^{b}\left(k_{3}\right) P_{R} u^{c}\left(k_{2}\right)\left(T^{a}\right)_{b c}, \\
& A_{4,5}=\left(\epsilon_{1}^{a} \cdot k_{3}\right)\left(\epsilon_{4}^{*} \cdot k_{2,1}\right) \bar{u}^{b}\left(k_{3}\right) P_{R} u^{c}\left(k_{2}\right)\left(T^{a}\right)_{b c}, \\
& A_{6,17}=\bar{u}^{b}\left(k_{3}\right) P_{R, L} \quad k_{1}^{a} \quad k_{4}^{*} u^{c}\left(k_{2}\right)\left(T^{a}\right)_{b c}, \\
& A_{7,27}=\bar{u}^{b}\left(k_{3}\right) P_{R, L} k_{1}^{a} k_{4}^{*} \quad k_{1} u^{c}\left(k_{2}\right)\left(T^{a}\right)_{b c}, \\
& A_{8,9}=\left(\epsilon_{1}^{a} \cdot k_{2,3}\right) \bar{u}^{b}\left(k_{3}\right) P_{R} k_{4}^{*} u^{c}\left(k_{2}\right)\left(T^{a}\right)_{b c}, \\
& A_{10,32}=\left(\epsilon_{1}^{a} \cdot \epsilon_{4}^{*}\right) \bar{u}^{b}\left(k_{3}\right) P_{R, L} \quad k_{1} u^{c}\left(k_{2}\right)\left(T^{a}\right)_{b c}, \\
& A_{11,12}=\left(\epsilon_{4}^{*} \cdot k_{2}\right)\left(\epsilon_{1}^{a} \cdot k_{2,3}\right) \bar{u}^{b}\left(k_{3}\right) P_{R} k_{1} u^{c}\left(k_{2}\right)\left(T^{a}\right)_{b c}, \\
& A_{13,14}=\left(\epsilon_{4}^{*} \cdot k_{1}\right)\left(\epsilon_{1}^{a} \cdot k_{2,3}\right) \bar{u}^{b}\left(k_{3}\right) P_{R} \quad k_{1} u^{c}\left(k_{2}\right)\left(T^{a}\right)_{b c}, \\
& A_{15,16}=\left(\epsilon_{4}^{*} \cdot k_{1,2}\right) \bar{u}^{b}\left(k_{3}\right) P_{R} k_{1}^{a} u^{c}\left(k_{2}\right)\left(T^{a}\right)_{b c}, \\
& A_{18,19}=\left(\epsilon_{4}^{*} \cdot k_{1,2}\right) \bar{u}^{b}\left(k_{3}\right) P_{R} k_{1}^{a} \quad k_{1} u^{c}\left(k_{2}\right)\left(T^{a}\right)_{b c}, \\
& A_{20,21}=\left(\epsilon_{1}^{a} \cdot k_{2}\right)\left(\epsilon_{4}^{*} \cdot k_{1,2}\right) \bar{u}^{b}\left(k_{3}\right) P_{L} u^{c}\left(k_{2}\right)\left(T^{a}\right)_{b c}, \\
& A_{23,24}=\left(\epsilon_{1}^{a} \cdot k_{3}\right)\left(\epsilon_{4}^{*} \cdot k_{2,1}\right) \bar{u}^{b}\left(k_{3}\right) P_{L} u^{c}\left(k_{2}\right)\left(T^{a}\right)_{b c}, \\
& A_{25,26}=\left(\epsilon_{4}^{*} \cdot k_{1,2}\right) \bar{u}^{b}\left(k_{3}\right) P_{L} k_{1}^{a} u^{c}\left(k_{2}\right)\left(T^{a}\right)_{b c}, \\
& A_{28,29}=\left(\epsilon_{4}^{*} \cdot k_{1,2}\right) \bar{u}^{b}\left(k_{3}\right) P_{L} k_{1}^{a} \quad k_{1} u^{c}\left(k_{2}\right)\left(T^{a}\right)_{b c}, \\
& A_{30,31}=\left(\epsilon_{1}^{a} \cdot k_{2,3}\right) \bar{u}^{b}\left(k_{3}\right) P_{L} k_{4}^{*} u^{c}\left(k_{2}\right)\left(T^{a}\right)_{b c}, \\
& A_{33,35}=\left(\epsilon_{4}^{*} \cdot k_{2,1}\right)\left(\epsilon_{1}^{a} \cdot k_{2}\right) \bar{u}^{b}\left(k_{3}\right) P_{L} \quad k_{1} u^{c}\left(k_{2}\right)\left(T^{a}\right)_{b c}, \\
& A_{34,36}=\left(\epsilon_{1}^{a} \cdot k_{3}\right)\left(\epsilon_{4}^{*} \cdot k_{2,1}\right) \bar{u}^{b}\left(k_{3}\right) P_{L} \quad k_{1} u^{c}\left(k_{2}\right)\left(T^{a}\right)_{b c}, \\
& A_{37,38}=\left(\epsilon_{1}^{a} \cdot k_{3,2}\right) \bar{u}^{b}\left(k_{3}\right) P_{L} k_{4}^{*} k_{1} u^{c}\left(k_{2}\right)\left(T^{a}\right)_{b c} .
\end{aligned}
$$

The relevant Feynman diagrams of the SUSY QCD corrected amplitude $M_{v i r}^{A}$ are shown 
in Fig, 3 , and $M_{v i r}^{A}$ can be written as

$$
M_{v i r}^{A}=M_{\text {self }}^{A}+M_{v e r t e x}^{A}+M_{b o x}^{A}
$$

where $M_{\text {self }}^{A}, M_{\text {vertex }}^{A}$ and $M_{b o x}^{A}$ come from self-energy diagrams, vertex diagrams and boxdiagrams as shown in Fig, 3 , respectively. Their explicit expressions are given by

$$
M_{\text {self }}^{A}=\sum_{m=1}^{38} f_{m}^{\text {self }} A_{m}, \quad M_{\text {vertex }}^{A}=\sum_{m=1}^{38} f_{m}^{v} A_{m}, \quad M_{b o x}^{A}=\sum_{m=1}^{38} f_{m}^{b} A_{m}
$$

where $f_{m}^{s e l f}, f_{m}^{v}, f_{m}^{b}$ are the form factors, which are given explicitly in Appendix.

The counterterms $M_{\text {count }}^{A}$, the corresponding diagrams of which are shown in Fig, 4 , can be written as follows

$$
M_{\text {count }}^{A}=\delta M_{\text {self }}^{A}+\delta M_{\text {vertex }}^{A}
$$

with

$$
\begin{aligned}
\delta M_{\text {self }}^{A}= & \delta M_{\text {self }}^{1}+\delta M_{\text {self }}^{2} \\
= & \frac{e g_{s} V_{t b}}{2 \sqrt{2} \sin \theta_{W}}\left\{\frac{A_{17}}{t-m_{t}^{2}}\left[m_{t}\left(\delta Z_{L}^{t t}-\delta Z_{R}^{t t}\right)+2 \delta m_{t}\right]-\frac{2 \delta Z_{L}^{b b}}{s}\left(A_{7}-2 A_{8}+2 A_{10}\right)\right. \\
& \left.+\frac{2}{\left(t-m_{t}^{2}\right)^{2}}\left(A_{7}+2 A_{9}-2 A_{15}\right)\left[\left(m_{t}^{2}-t\right) \delta Z_{L}^{t t}+2 m_{t} \delta m_{t}\right]\right\} \\
\delta M_{\text {vertex }}^{A}= & \sum_{n=1}^{4} \delta M_{\text {vertex }}^{n}, \\
\delta M_{\text {vertex }}^{1}= & \frac{e g_{s} V_{t b}}{2 \sqrt{2} s \sin \theta_{W}}\left(A_{7}+2 A_{8}-2 A_{10}\right)\left(\delta Z_{L}^{b b}+\delta Z_{L}^{t t}\right), \\
\delta M_{\text {vertex }}^{2}= & \frac{e g_{s} V_{t b}}{2 \sqrt{2} \sin \theta_{W}\left(t-m_{t}^{2}\right)}\left(A_{7}+2 A_{9}-2 A_{15}\right)\left(\delta Z_{L}^{b b}+\delta Z_{L}^{t t}\right), \\
\delta M_{\text {vertex }}^{3}= & \frac{e g_{s} V_{t b}}{2 \sqrt{2} \sin \theta_{W}\left(t-m_{t}^{2}\right)}\left[2 m_{t} A_{17}\left(\delta Z_{R}^{t t}-\delta Z_{L}^{t t}\right)\right. \\
\delta M_{\text {vertex }}^{4}= & \frac{e g_{s} V_{t b}}{2 \sqrt{2} s \sin \theta_{W}}\left(2 \delta Z_{g}+\delta Z_{A A}+2 \delta Z_{L}^{b b}\right)\left(A_{7}+2 A_{8}-2 A_{10}\right) .
\end{aligned}
$$

The partonic cross section can be written as

$$
\hat{\sigma}=\int_{-1}^{1} \mathrm{~d} z \frac{1}{32 \pi s^{2}} \lambda^{1 / 2} \overline{\left|M_{r e n}^{A}\right|^{2}}=\int_{t_{-}}^{t_{+}} \mathrm{d} t \frac{1}{16 \pi s^{2}} \overline{\left|M_{r e n}^{A}\right|^{2}}
$$

where $\lambda \equiv\left(s-m_{t}^{2}+m_{W}^{2}\right)^{2}-4 s m_{W}^{2}, t_{ \pm}=\frac{1}{2}\left[m_{t}^{2}+m_{W}^{2}-s \pm \lambda^{1 / 2}\right]$, and $\overline{\left|M_{\text {ren }}^{A}\right|^{2}}$ is the renormalized amplitude squared given by

$$
\overline{\left|M_{r e n}^{A}\right|^{2}}=\bar{\sum}\left|M_{0}^{A}\right|^{2}+2 \operatorname{Re} \bar{\sum} M_{0}^{A}\left[M_{v i r}^{A}+M_{\text {count }}^{A}\right]^{\dagger},
$$


where the colors and spins of the outgoing particles have been summed over, and the colors and spins of incoming ones have been averaged over.

The total cross section at the LHC is obtained by convoluting the partonic cross section with the parton distribution functions (PDFs) $G_{g, b / p}$ in the proton:

$$
\sigma=\int_{\tau_{0}}^{1} \mathrm{~d} x_{1} \int_{\tau_{0} / x_{1}}^{1} \mathrm{~d} x_{2}\left[G_{g / p}\left(x_{1}, \mu_{f}\right) G_{b / p}\left(x_{2}, \mu_{f}\right)+\left(x_{1} \leftrightarrow x_{2}\right)\right] \hat{\sigma}(\tau S),
$$

where $\mu_{f}$ is the factorization scale and $S=\left(P_{1}+P_{2}\right)^{2}, P_{1}$ and $P_{2}$ are the four-momentum of the incident hadrons, $\tau_{0}=\frac{\left(m_{W}+m_{t}\right)^{2}}{S}, \tau=x_{1} x_{2}$, and $x_{1}, x_{2}$ are the longitudinal momentum fractions of initial partons in the hadrons.

\section{B. s-channel and t-channel}

For convenience, we first define the reduced standard matrix elements $F_{i}$ as follows:

$$
\begin{aligned}
F_{1,2} & =\bar{v}\left(k_{2}\right) P_{R, L} \gamma^{\mu} u\left(k_{1}\right) \bar{u}\left(k_{3}\right) P_{R} \gamma_{\mu} v\left(k_{4}\right), \\
F_{3,4} & =\bar{v}\left(k_{2}\right) P_{R, L} u\left(k_{1}\right) \bar{u}\left(k_{3}\right) P_{R} k_{1} v\left(k_{4}\right), \\
F_{5,6} & =\bar{u}\left(k_{3}\right) P_{R, L} v\left(k_{4}\right) \bar{v}\left(k_{2}\right) P_{R} k_{3} u\left(k_{1}\right), \\
F_{7,8} & =\bar{v}\left(k_{2}\right) P_{R, L} u\left(k_{1}\right) \bar{u}\left(k_{3}\right) P_{L} v\left(k_{4}\right), \\
F_{9} & =\bar{v}\left(k_{2}\right) P_{R} \gamma^{\mu} u\left(k_{1}\right) \bar{u}\left(k_{3}\right) P_{L} \gamma_{\mu} v\left(k_{4}\right),
\end{aligned}
$$

which will appear in the amplitudes of s-channel and t-channel below.

For s-channel, the diagrams which contribute to the LO amplitude $M_{0}^{s}$ are shown in Fig,2 (c). The LO amplitude $M_{0}^{s}$ is

$$
M_{0}^{s}=-\sum_{\substack{q=u, c \\ q^{\prime}=d, s, b}} \frac{2 \pi \alpha V_{t b} V_{q q^{\prime}}^{*}}{\sin ^{2} \theta_{W}\left(s-m_{W}^{2}\right)} F_{1},
$$

The virtual corrections $M_{v i r}^{s}$ contains the radiative corrections from the one-loop vertex diagrams, which are shown in Fig 5 (a) and (b), and we can write $M_{v i r}^{s}$ as:

$$
M_{v i r}^{s}=\sum_{m=1}^{9} f_{m}^{s} F_{m}
$$

where $f_{m}^{s}(m=1,2, \cdots, 9)$ are form factors, which are given explicitly in Appendix.

The corresponding counterterm can be written as

$$
M_{\text {count }}^{s}=-\sum_{\substack{q=u, c \\ q^{\prime}=d, s, b}} \frac{\pi \alpha V_{t b} V_{q q^{\prime}}^{*}}{\sin ^{2} \theta_{W}\left(s-m_{W}^{2}\right)}\left(\delta Z_{L}^{b b}+\delta Z_{L}^{t t}+\delta Z_{L}^{q q}+\delta Z_{L}^{q^{\prime} q^{\prime}}\right) F_{1},
$$


where the expressions of $\delta Z_{L}^{i i}(i=u, d, s, c, b, t)$ are shown in Eq.(2).

According to the crossing symmetry, we have the similar expressions in t-channel as in s-channel. We replace the variable $s$ by $t$ in Eq.(17), (18) and (19), then use the different summation over quark flavors, and change the indices of the quarks in the initial and final states. For example,

$$
\begin{aligned}
M_{0}^{t} & =M_{0}^{s}\left(s \rightarrow t, \sum_{q, q^{\prime}} \rightarrow \sum_{\left\{q q^{\prime}\right\}}\right)=-\sum_{\left\{q q^{\prime}\right\}} \frac{4 \pi \alpha V_{t b} V_{q q^{\prime}}^{*}}{\sin ^{2} \theta_{W}\left(t-m_{W}^{2}\right)} F_{1}^{\prime}, \\
M_{v i r}^{t} & =M_{v i r}^{s}\left(s \rightarrow t, \sum_{q, q^{\prime}} \rightarrow \sum_{\left\{q q^{\prime}\right\}}\right)=\sum_{m=1}^{9} f_{m}^{t}\left(s \rightarrow t, \sum_{q, q^{\prime}} \rightarrow \sum_{\left\{q q^{\prime}\right\}}\right) F_{m}^{\prime} \\
M_{\text {count }}^{t} & =M_{\text {count }}^{s}\left(s \rightarrow t, \sum_{q, q^{\prime}} \rightarrow \sum_{\left\{q q^{\prime}\right\}}\right) \\
& =-\sum_{\left\{q q^{\prime}\right\}} \frac{2 \pi \alpha V_{t b} V_{q q^{\prime}}^{*}}{\sin ^{2} \theta_{W}\left(t-m_{W}^{2}\right)}\left(\delta Z_{L}^{q q}+\delta Z_{L}^{q^{\prime} q^{\prime}}+\delta Z_{L}^{b b}+\delta Z_{L}^{t t}\right) F_{1}^{\prime},
\end{aligned}
$$

with

$$
F_{m}^{\prime} \equiv F_{m}\left(v\left(k_{4}\right) \rightarrow u\left(k_{2}\right), \bar{v}\left(k_{2}\right) \rightarrow u\left(k_{3}\right)\right)
$$

Here the index pair $\left\{q q^{\prime}\right\}$ takes on the flavors $\{u d, u s, u b, c d, c s, c b\}$. The other formulas for cross sections are the same as in associated production.

\section{NUMERICAL RESULTS AND DISCUSSIONS}

In this section, we present the numerical results for the SUSY QCD corrections to the three channels of single top production at the LHC. For comparison, we also present the numerical results for s-channel at the Tevatron[32]. In our numerical calculations, we use the following set of the SM parameters [35]]:

$$
m_{t}=175 \mathrm{GeV}, \quad \alpha_{e w}\left(M_{W}\right)=1 / 128, \quad \alpha_{s}\left(M_{Z}\right)=0.118,
$$

and all light quark masses are set to be zero, and the CKM matrix elements are taken to be the values shown in Ref. 35$]$.

The running QCD coupling $\alpha_{s}(Q)$ is evaluated at two-loop order[36], and the CTEQ6M PDFs[37] are used throughout this paper to calculate cross sections. For simplicity, we 
neglect the b-quark mass. We choose $\mu_{r}=\mu_{f}=m_{t}+m_{W}$ for the renormalization and factorization scales in associated production and choose $\mu_{r}=\mu_{f}=m_{t}$ for the renormalization and factorization scales in the other two channels.

Besides, the values of the MSSM parameters taken in our numerical calculations are constrained within the minimal supergravity scenario(mSUGRA) [38], in which there are only five free input parameters at the grand unification where $M_{1 / 2}, M_{0}, A_{0}, \tan \beta$ and the sign of $\mu$, where $M_{1 / 2}, M_{0}, A_{0}$ are, respectively, the universal gaugino mass, scalar mass, and the trilinear soft breaking parameter in the superpotential. Given these parameters, all the MSSM parameters at the weak scale are determined in the mSUGRA scenario by using the program package SUSPECT 2.3 [39], where we set $A_{0}=-200 \mathrm{GeV}$ and $\mu>0$.

\section{A. Associated Production}

We define the K factor as the ratio of the SUSY QCD corrected cross sections to LO total cross sections, calculated using the CTEQ6M PDFs. Fig [6 shows the K factors as functions of $M_{\tilde{g}}\left(M_{1 / 2}\right)$ for the associated production process $p p \rightarrow t W$ at the LHC for $\tan \beta=5,20$ and 35, respectively. From Fig, we can see that the differences among the results are small for different $\tan \beta$, and $\mathrm{K}$ factors increase with the increasing $M_{\tilde{g}}$ for small $M_{\tilde{g}}(\lesssim 160 \mathrm{GeV})$, while decrease with the increasing $M_{\tilde{g}}$ for large $M_{\tilde{g}}(\gtrsim 160 \mathrm{GeV})$, and, in general, the $\mathrm{K}$ factors are about 1.06 .

In Fig:7 we show the dependence of the $\mathrm{K}$ factors on $M_{\tilde{t}_{1}}\left(M_{0}\right)$ for different $\tan \beta$. Fig:7 shows that the $\mathrm{K}$ factors have the similar behaviors as those shown in Fig 6 , and are also about 1.06 in general.

To compare with Fig:7, we present Fig 10 which takes the similar parameters as those used in Fig:7 but $M_{1 / 2}=70 \mathrm{GeV}$, where the gluino mass $M_{\tilde{g}}$ lies in the range $220 \mathrm{GeV} \lesssim$ $M_{\tilde{g}} \lesssim 250 \mathrm{GeV}$ for all values of $M_{0}$ and $\tan \beta$ we assumed here. Fig 10 shows that SUSY QCD corrections are not sensitive to $\tan \beta$, which is consistent with Fig.7

Fig 8 gives the $\mathrm{K}$ factors as functions of $M_{\tilde{g}}\left(M_{1 / 2}\right)$ for different $M_{0}$, assuming $\tan \beta=$ 5. In Fig 8 we can see that there are large differences between different $M_{0}$ when $M_{\tilde{g}}$ becomes small, but these curves approach each other when $M_{\tilde{g}}$ becomes large because of the decoupling of heavy gluino $\left(M_{\tilde{g}} \gtrsim 450 \mathrm{GeV}\right)$. The $\mathrm{K}$ factors are about 1.06 for $M_{\tilde{g}} \lesssim 500 \mathrm{GeV}$, and become small slowly with increasing $M_{\tilde{g}}$, but the $\mathrm{K}$ factors decrease rapidly when 
$M_{\tilde{g}} \lesssim 150 \mathrm{GeV}$ for $M_{0}=150 \mathrm{GeV}$.

In Fig 9 we present the $\mathrm{K}$ factors as functions of $M_{\tilde{t}_{1}}\left(M_{0}\right)$, assuming $\tan \beta=5$, and $M_{1 / 2}=40,70$ and $100 \mathrm{GeV}$, respectively. From Fig.9, we find the similar results as those shown in Fig, 8 , i.e. the $\mathrm{K}$ factors are about 1.06 for most values of $M_{\tilde{t}_{1}}$ considered, and become small slowly with increasing $M_{\tilde{t}_{1}}$.

In Fig 11 we present the LO and the SUSY QCD corrected cross sections as functions of renormalization and factorization scales $\mu / \mu_{0}\left(\mu_{f}=\mu_{r}=\mu, \mu_{0}=m_{t}+m_{W}\right)$, respectively, assuming $\tan \beta=5, M_{0}=150 \mathrm{GeV}, M_{1 / 2}=70 \mathrm{GeV}, A_{0}=-200 \mathrm{GeV}$ and $\mu>0$. This figure shows that the scale dependence of the SUSY QCD corrected total cross section is a little larger than that of the LO cross section because of the running effects of the extra $\alpha_{s}$ in SUSY QCD corrections. We can recover the LO results of scale dependence by dividing $\alpha_{s}$ in the SUSY QCD corrections. After comparison with the NLO QCD corrections[28], we can see that if the NLO QCD corrections are also included, $\mathcal{O}\left(\alpha_{s}\right)$ corrections still improve the scale dependence.

\section{B. s-channel and t-channel}

For the s-channel process $p p \rightarrow t \bar{b}$, in Fig 12 (a)-(b), we display the $\mathrm{K}$ factors as functions of $M_{\tilde{g}}\left(M_{1 / 2}\right)$ and $M_{\tilde{t}_{1}}\left(M_{0}\right)$, respectively, assuming $M_{0}=150,300 \mathrm{GeV}$ and $M_{1 / 2}=$ 40,70, 100GeV. Fig 12 shows that the K factors are about 1.01 for some favorable parameters, otherwise, the $\mathrm{K}$ factors approach the unit value.

In Fig,13 (a)-(b), the $\mathrm{K}$ factors are plotted as functions of $M_{\tilde{g}}\left(M_{1 / 2}\right)$ and $M_{\tilde{t}_{1}}\left(M_{0}\right)$, respectively, assuming $\tan \beta=5,20,35$. Fig 13 shows that the $K$ factors can reach 1.01 for light $M_{\tilde{g}}$ and $M_{\tilde{t}_{1}}$, respectively, and are not sensitive to $\tan \beta$.

In Fig 14, we display the $\mathrm{K}$ factors as functions of $M_{\tilde{t}_{1}}$ for the s-channel process $p \bar{p} \rightarrow t \bar{b}$ at the Tevatron Run II. We find that the $\mathrm{K}$ factors can reach 1.01 for small $M_{\tilde{t}_{1}}$, and decrease rapidly as $M_{\tilde{t}_{1}}$ increases. These results are consistent with that shown in Fig.3 of Ref.[32], where the relevant parameters assumed are the same as ones used in our numerical calculations.

For the t-channel process $p p \rightarrow q t$ at the LHC, Fig 15 shows the K factors as functions of $M_{\tilde{g}}$ for $\tan \beta=5$ and $M_{0}=150,300 \mathrm{GeV}$, respectively. From Fig.15, we find that the SUSY QCD corrections decrease the total cross sections and the $\mathrm{K}$ factors approach the unit value 
for all parameters assumed here, which means that SUSY QCD corrections are negligible.

In Fig 16 and Fig 17 we present the LO and the SUSY QCD corrected cross sections as functions of renormalization and factorization scales $\mu / \mu_{0}\left(\mu_{f}=\mu_{r}=\mu, \mu_{0}=m_{t}\right)$ for both s-channel and t-channel, respectively, assuming $\tan \beta=5, M_{0}=150 \mathrm{GeV}, M_{1 / 2}=70 \mathrm{GeV}$, $A_{0}=-200 \mathrm{GeV}$ and $\mu>0$. Since the SUSY QCD corrections to the two channels are very small, they do not affect the scale dependence of the LO results obviously.

\section{CONCLUSION}

In conclusion, we have calculated the SUSY QCD corrections to the total cross sections for single top production at the Tevatron and the LHC in the MSSM. Our results show that for the s-channel and t-channel, the SUSY QCD corrections are at most about 1\%, but for the associated production process $p p \rightarrow t W$, the SUSY QCD corrections increase the total cross sections significantly, which can reach about $6 \%$ for most values of the parameters, and the SUSY QCD corrections should be taken into consideration in the future high precision experimental analysis for top physics at the LHC.

\section{Acknowledgments}

This work was supported in part by the National Natural Science Foundation of China, under grants No. 10421503 and No. 10575001, and the Key Grant Project of Chinese Ministry of Education under grant No. 305001.

\section{Appendix}

In this Appendix, we will list the explicit expressions of the non-zero form factors of associated production and s-channel. For simplicity, we first define the abbreviations for Passarino-Veltman functions [42] below.

$$
\begin{gathered}
B_{0}^{s}=B_{0}\left(s, M_{\tilde{g}}^{2}, M_{\tilde{t}_{i}}^{2}\right), \quad B_{1}^{s}=B_{1}\left(s, M_{\tilde{g}}^{2}, M_{\tilde{t}_{i}}^{2}\right), \quad B_{0}^{\prime s}=B_{0}\left(s, M_{\tilde{g}}^{2}, M_{\tilde{b}_{i}}^{2}\right), \\
B_{0}^{t}=B_{0}\left(t, M_{\tilde{g}}^{2}, M_{\tilde{t}_{i}}^{2}\right), \quad B_{1}^{t}=B_{1}\left(t, M_{\tilde{g}}^{2}, M_{\tilde{t}_{i}}^{2}\right), \\
C_{i j k \cdots}^{a}=C_{i j k \cdots}\left(m_{t}^{2}, s, m_{W}^{2}, M_{\tilde{t}_{i}}^{2}, M_{\tilde{g}}^{2}, M_{\tilde{b}_{j}}^{2}\right),
\end{gathered}
$$




$$
\begin{gathered}
C_{i j k \cdots}^{b}=C_{i j k \cdots}\left(0, t, m_{W}^{2}, M_{\tilde{b}_{i}}^{2}, M_{\tilde{g}}^{2}, M_{\tilde{t}_{j}}^{2}\right), \\
C_{i j k \cdots}^{c}=C_{i j k \cdots}\left(m_{t}^{2}, t, 0, M_{\tilde{g}}^{2}, M_{\tilde{t}_{i}}^{2}, M_{\tilde{g}}^{2}\right), \\
C_{i j k \cdots}^{d}=C_{i j k \cdots}\left(m_{t}^{2}, t, 0, M_{\tilde{t}_{i}}^{2}, M_{\tilde{g}}^{2}, M_{\tilde{t}_{i}}^{2}\right), \\
C_{i j k \cdots}^{e}=C_{i j k \cdots}\left(m_{t}^{2}, t, 0, M_{\tilde{g}}^{2}, M_{\tilde{t}_{i}}^{2}, M_{\tilde{t}_{i}}^{2}\right), \\
C_{i j k \cdots}^{f}=C_{i j k \cdots}\left(0, s, 0, M_{\tilde{g}}^{2}, M_{\tilde{b}_{i}}^{2}, M_{\tilde{g}}^{2}\right), \\
C_{i j k \cdots}^{g}=C_{i j k \cdots}\left(0, s, 0, M_{\tilde{b}_{i}}^{2}, M_{\tilde{g}}^{2}, M_{\tilde{b}_{i}}^{2}\right), \\
C_{i j k \cdots}^{i}=C_{i j k \cdots(}=C_{i j k \cdots}\left(m_{W}^{2}, m_{t}^{2}, s, M_{\tilde{b}_{i}}^{2}, M_{\tilde{t}_{j}}^{2}, M_{\tilde{g}}^{2}\right), \\
\left.C_{i j k \cdots}^{A}=C_{i j k \cdots}^{2}, M_{\tilde{b}_{i}}^{2}, M_{\tilde{t}_{j}}^{2}\right), \\
C_{i j k \cdots}^{B}=C_{i j k \cdots}\left(m_{t}^{2}, s, 0, M_{\tilde{g}}^{2}, M_{\tilde{u}_{i}}^{2}, M_{\tilde{d}_{j}}^{2}\right), \\
\left.D_{i j k \cdots}^{a}=D_{i j k \cdots}^{2}, M_{\tilde{t}_{i}}^{2}, M_{\tilde{b}_{j}}^{2}\right), \\
\left.D_{i j k \cdots}^{b}=D_{i j k \cdots}\left(0, m_{W}^{2}, m_{t}^{2}, 0, t, s, M_{\tilde{g}}^{2}, M_{\tilde{b}_{i}}^{2}, M_{\tilde{t}_{j}}^{2}, M_{\tilde{g}}^{2}\right), u, 0, m_{t}^{2}, M_{\tilde{b}_{i}}^{2}, M_{\tilde{g}}^{2}, M_{\tilde{b}_{i}}^{2}, M_{\tilde{t}_{j}}^{2}\right), \\
D_{i j k \cdots}^{c}=D_{i j k \cdots}\left(m_{t}^{2}, t, m_{W}^{2}, u, 0,0, M_{\tilde{t}_{i}}^{2}, M_{\tilde{g}}^{2}, M_{\tilde{t}_{i}}^{2}, M_{\tilde{b}_{j}}^{2}\right) .
\end{gathered}
$$

For s-channel, we have

$$
\begin{gathered}
f_{1}^{s}=-\sum_{\substack{q=u, c \\
q^{\prime}=d, s, b}} \sum_{\substack{i, j=1 \\
\sin ^{2} \theta_{W}\left(s-m_{W}^{2}\right)}}^{2}\left(U_{i 1}^{q} U_{j 1}^{q^{\prime}} U_{i 1}^{q *} U_{j 1}^{q^{\prime} *} C_{00}^{A}+U_{i 1}^{t} U_{j 1}^{b} U_{i 1}^{t *} U_{j 1}^{b *} C_{00}^{B}\right), \\
f_{2}^{s}=-\sum_{\substack{q=u, c \\
q^{\prime}=d, s, b}} \sum_{i, j=1}^{2} \frac{8 \alpha \alpha_{s} V_{t b} V_{q q^{\prime}}^{*}}{3 \sin ^{2} \theta_{W}\left(s-m_{W}^{2}\right)} U_{i 2}^{q} U_{j 1}^{q^{\prime}} U_{i 1}^{q *} U_{j 2}^{q^{\prime} *} C_{00}^{A}, \\
f_{3}^{s=-} \sum_{\substack{q=u, c \\
q^{\prime}=d, s, b}} \sum_{i, j=1}^{2} \frac{8 \alpha \alpha_{s} V_{t b} V_{q q^{\prime}}^{*}}{3 \sin ^{2} \theta_{W}\left(s-m_{W}^{2}\right)} M_{\tilde{g}} U_{i 2}^{q} U_{j 1}^{q^{\prime}} U_{i 1}^{q *} U_{j 1}^{q^{\prime} *}\left(C_{0}^{A}+C_{1}^{A}+C_{2}^{A}\right), \\
f_{4}^{s=-} \sum_{\substack{q=u, c \\
q^{\prime}=d, s, b}} \sum_{i, j=1}^{2} \frac{8 \alpha \alpha_{s} V_{t b} V_{q q^{\prime}}^{*}}{3 \sin ^{2} \theta_{W}\left(s-m_{W}^{2}\right)} M_{\tilde{g}} U_{i 1}^{q} U_{j 1}^{q^{\prime}} U_{i 1}^{q *} U_{j 2}^{q^{\prime} *}\left(C_{0}^{A}+C_{1}^{A}+C_{2}^{A}\right), \\
f_{5}^{s}=-\sum_{\substack{q=u, c \\
q^{\prime}=d, s, b}} \sum_{i, j=1}^{2} \frac{8 \alpha \alpha_{s} V_{t b} V_{q q^{\prime}}^{*}}{3 \sin ^{2} \theta_{W}\left(s-m_{W}^{2}\right)}\left[M_{\tilde{g}} U_{i 1}^{t} U_{j 2}^{b} U_{i 1}^{t *} U_{j 1}^{b *}\left(C_{0}^{B}+C_{1}^{B}+C_{2}^{B}\right)\right. \\
\left.+m_{t} U_{i 1}^{t} U_{j 2}^{b} U_{i 2}^{t *} U_{j 1}^{b *}\left(C_{1}^{B}+C_{11}^{B}+C_{12}^{B}\right)\right],
\end{gathered}
$$




$$
\begin{gathered}
f_{6}^{s}=-\sum_{\substack{q=u, c \\
q^{\prime}=d, s, b}} \sum_{i, j=1}^{2} \frac{8 \alpha \alpha_{s} V_{t b} V_{q q^{\prime}}^{*}}{3 \sin ^{2} \theta_{W}\left(s-m_{W}^{2}\right)}\left[m_{t} U_{i 1}^{t} U_{j 1}^{b} U_{i 1}^{t *} U_{j 1}^{b *}\left(C_{1}^{B}+C_{11}^{B}+C_{12}^{B}\right)\right. \\
\left.+M_{\tilde{g}} U_{i 1}^{t} U_{j 1}^{b} U_{i 2}^{t *} U_{j 1}^{b *}\left(C_{0}^{B}+C_{1}^{B}+C_{2}^{B}\right)\right], \\
f_{7}^{s}=\sum_{\substack{q=u, c \\
q^{\prime}=d, s, b}} \sum_{i, j=1}^{2} \frac{4 \alpha \alpha_{s} V_{t b} V_{q q^{\prime}}^{*}}{3 \sin ^{2} \theta_{W}\left(s-m_{W}^{2}\right)} m_{t} M_{\tilde{g}} U_{i 2}^{q} U_{j 1}^{q^{\prime}} U_{i 1}^{q *} U_{j 1}^{q^{\prime} *}\left(C_{0}^{A}+2 C_{2}^{A}\right), \\
f_{8}^{s}=\sum_{\substack{q=u, c \\
q^{\prime}=d, s, b}} \sum_{i, j=1}^{2} \frac{4 \alpha \alpha_{s} V_{t b} V_{q q^{\prime}}^{*}}{3 \sin ^{2} \theta_{W}\left(s-m_{W}^{2}\right)} m_{t} M_{\tilde{g}} U_{i 1}^{q} U_{j 1}^{q^{\prime}} U_{i 1}^{q *} U_{j 2}^{q^{\prime} *}\left(C_{0}^{A}+2 C_{2}^{A}\right), \\
f_{9}^{s}=-\sum_{\substack{q=u, c \\
q^{\prime}=d, s, b}} \sum_{i, j=1}^{2} \frac{8 \alpha \alpha_{s} V_{t b} V_{q q^{\prime}}^{*}}{3 \sin ^{2} \theta_{W}\left(s-m_{W}^{2}\right)} C_{00}^{B} U_{i 1}^{t} U_{j 2}^{b} U_{i 2}^{t *} U_{j 1}^{b *} .
\end{gathered}
$$

For associated production, we calculate the individual diagram separately by different types, as shown in Fig 3 and Eq.(18). The form factors of the self-energy diagrams are

$$
\begin{gathered}
f_{1}^{s e l f}=-\frac{2 \sqrt{2} \alpha_{s} g_{s} e V_{t b}}{3 \pi s \sin \theta_{W}} \sum_{i}^{2} M_{\tilde{g}} U_{i 2}^{b} U_{i 1}^{b *} B_{0}^{s}, \\
f_{7}^{\text {self }}=\frac{\sqrt{2} \alpha_{s} g_{s} e V_{t b}}{3 \pi \sin \theta_{W}} \sum_{i}^{2}\left\{\frac{1}{s} U_{i 1}^{b} U_{i 1}^{b *} B_{1}^{s}-\frac{1}{\left(t-m_{t}^{2}\right)^{2}}\left[M_{\tilde{g}} m_{t}\left(U_{i 2}^{t} U_{i 1}^{t *}+U_{i 1}^{t} U_{i 2}^{t *}\right) B_{0}^{t}\right.\right. \\
\left.\left.+\left(t U_{i 1}^{t} U_{i 1}^{t *}+m_{t}^{2} U_{i 2}^{t} U_{i 2}^{t *}\right) B_{1}^{t}\right]\right\} \\
f_{8}^{s e l f}=\frac{2 \sqrt{2} \alpha_{s} g_{s} e V_{t b}}{3 \pi s \sin \theta_{W}} \sum_{i}^{2} U_{i 1}^{b} U_{i 1}^{b *} B_{1}^{s}, \\
f_{9}^{s e l f}=-f_{15}^{s e l f}=-\frac{2 \sqrt{2} \alpha_{s} g_{s} e V_{t b}}{3 \pi \sin \theta_{W}\left(t-m_{t}^{2}\right)^{2}} \sum_{i}^{2}\left[M_{\tilde{g}} m_{t}\left(U_{i 2}^{t} U_{i 1}^{t *}+U_{i 1}^{t} U_{i 2}^{t *}\right) B_{0}^{t}+\left(t U_{i 1}^{t} U_{i 1}^{t *}+m_{t}^{2} U_{i 2}^{t} U_{i 2}^{t *}\right) B_{1}^{t}\right], \\
f_{10}^{s e l f}=\frac{\sqrt{2} \alpha_{s} g_{s} e V_{t b}}{3 \pi s \sin \theta_{W}} \sum_{i}^{2}\left[M_{\tilde{g}} U_{i 2}^{b} U_{i 1}^{b *} B_{0}^{s}+2 U_{i 1}^{b} U_{i 1}^{b *} B_{1}^{s}\right], \\
f_{17}^{s e l f}=\frac{2}{3 \pi \sin \theta_{W}\left(t-m_{t}^{2}\right)^{2}} \sum_{i}\left[M_{\tilde{g}}\left(m_{t}^{2} U_{i 1}^{t} U_{i 2}^{t *}-t U_{i 1}^{t} U_{i 2}^{t *}\right) B_{0}^{t}+m_{t}\left(m_{t}^{2} U_{i 2}^{t} U_{i 2}^{t *}-t U_{i 2}^{t} U_{i 2}^{t *}\right) B_{1}^{t}\right] .
\end{gathered}
$$

To avoid the very long expressions, we define

$$
f_{m}^{v} \equiv \sum_{\alpha=1}^{7} f_{m}^{v \alpha}
$$

as shown in Fig 3 , where

$$
f_{2}^{v 1}=f_{3}^{v 1}=-\sum_{i}^{2} \sum_{j}^{2} \frac{4 \sqrt{2} \alpha_{s} e g_{s} V_{t b} U_{i 1}^{t} U_{j 1}^{b *}}{3 s \pi \sin \theta_{W}}\left[m_{t} U_{j 2}^{b} U_{i 2}^{t *}\left(C_{1}^{a}+C_{11}^{a}+C_{12}^{a}\right)-M_{\tilde{g}} U_{j 2}^{b} U_{i 1}^{t *} C_{1}^{a}\right],
$$




$$
\begin{aligned}
& f_{7}^{v 1}=\frac{1}{2} f_{8}^{v 1}=-\frac{1}{2} f_{10}^{v 1}=-\sum_{i}^{2} \sum_{j}^{2} \frac{4 \sqrt{2} \alpha_{s} e g_{s} V_{t b} U_{i 1}^{t} U_{j 1}^{b *}}{3 s \pi \sin \theta_{W}} U_{j 1}^{b} U_{i 1}^{t *} C_{00}^{a} \\
& f_{15}^{v 1}=f_{16}^{v 1}=-\sum_{i}^{2} \sum_{j}^{2} \frac{2 \sqrt{2} \alpha_{s} e g_{s} V_{t b} U_{i 1}^{t} U_{j 1}^{b *}}{3 \pi \sin \theta_{W}} U_{j 1}^{b} U_{i 1}^{t *} C_{12}^{a} \\
& f_{18}^{v 1}=f_{19}^{v 1}=-\sum_{i}^{2} \sum_{j}^{2} \frac{2 \sqrt{2} \alpha_{s} e g_{s} V_{t b} U_{i 1}^{t} U_{j 1}^{b *}}{3 s \pi \sin \theta_{W}}\left[M_{\tilde{g}} U_{j 1}^{b} U_{i 2}^{t *} C_{1}^{a}-m_{t} U_{j 2}^{b} U_{i 2}^{t *}\left(C_{1}^{a}+C_{11}^{a}-C_{12}^{a}\right)\right] \\
& f_{20}^{v 1}=f_{21}^{v 1}=-\sum_{i}^{2} \sum_{j}^{2} \frac{4 \sqrt{2} \alpha_{s} e g_{s} V_{t b} U_{i 1}^{t} U_{j 1}^{b *}}{3 s \pi \sin \theta_{W}}\left[m_{t} U_{j 1}^{b} U_{i 1}^{t *}\left(C_{1}^{a}+C_{11}^{a}+C_{12}^{a}\right)-M_{\tilde{g}} U_{j 2}^{b} U_{i 1}^{t *} C_{1}^{a}\right] \\
& f_{25}^{v 1}=f_{26}^{v 1}=\sum_{i}^{2} \sum_{j}^{2} \frac{2 \sqrt{2} \alpha_{s} e g_{s} V_{t b} U_{i 1}^{t} U_{j 1}^{b *}}{3 \pi \sin \theta_{W}} U_{j 2}^{b} U_{i 2}^{t *} C_{12}^{a} \\
& f_{27}^{v 1}=\frac{1}{2} f_{30}^{v 1}=-\frac{1}{2} f_{32}^{v 1}=-\sum_{i}^{2} \sum_{j}^{2} \frac{2 \sqrt{2} \alpha_{s} e g_{s} V_{t b} U_{i 1}^{t} U_{j 1}^{b *}}{3 s \pi \sin \theta_{W}} U_{j 2}^{b} U_{i 2}^{t *} C_{00}^{a} \\
& f_{28}^{v 1}=f_{29}^{v 1}=-\sum_{i}^{2} \sum_{j}^{2} \frac{2 \sqrt{2} \alpha_{s} e g_{s} V_{t b} U_{i 1}^{t} U_{j 1}^{b *}}{3 s \pi \sin \theta_{W}}\left[M_{\tilde{g}} U_{j 1}^{b} U_{i 2}^{t *} C_{1}^{a}-m_{t} U_{j 1}^{b} U_{i 1}^{t *}\left(C_{1}^{a}+C_{11}^{a}+C_{12}^{a}\right)\right], \\
& f_{4}^{v 2}=-2 f_{19}^{v 2}=\frac{4 \sqrt{2} \alpha_{s} e g_{s} V_{t b}}{3 \pi \sin \theta_{W}\left(t-m_{t}^{2}\right)} \sum_{i}^{2} \sum_{j}^{2} U_{j 1}^{t} U_{i 1}^{b *}\left(m_{t} U_{i 2}^{b} U_{j 2}^{t *} C_{12}^{b}+M_{\tilde{g}} U_{i 2}^{b} U_{j 1}^{t *} C_{1}^{b}\right), \\
& f_{7}^{v 2}=\frac{1}{2} f_{9}^{v 2}=-\frac{1}{2} f_{15}^{v 2}=-\frac{2 \sqrt{2} \alpha_{s} e g_{s} V_{t b}}{3 \pi \sin \theta_{W}\left(t-m_{t}^{2}\right)} \sum_{i}^{2} \sum_{j}^{2} U_{j 1}^{t} U_{i 1}^{b *} U_{i 1}^{b} U_{j 1}^{t *} C_{00}^{b}, \\
& f_{16}^{v 2}=\frac{2 \sqrt{2} \alpha_{s} e g_{s} V_{t b}}{3 \pi \sin \theta_{W}} \sum_{i}^{2} \sum_{j}^{2} U_{j 1}^{t} U_{i 1}^{b *} U_{i 1}^{b} U_{j 1}^{t *} C_{12}^{b} \\
& f_{23}^{v 2}=-2 f_{29}^{v 2}=\frac{4 \sqrt{2} \alpha_{s} e g_{s} V_{t b}}{3 \pi \sin \theta_{W}\left(t-m_{t}^{2}\right)} \sum_{i}^{2} \sum_{j}^{2} U_{j 1}^{t} U_{i 1}^{b *}\left(m_{t} U_{i 1}^{b} U_{j 1}^{t *} C_{12}^{b}+M_{\tilde{g}} U_{i 1}^{b} U_{j 2}^{t *} C_{1}^{b}\right), \\
& f_{25}^{v 2}=-2 f_{27}^{v 2}=-f_{31}^{v 2}=\frac{4 \sqrt{2} \alpha_{s} e g_{s} V_{t b}}{3 \pi \sin \theta_{W}\left(t-m_{t}^{2}\right)} \sum_{i}^{2} \sum_{j}^{2} U_{j 1}^{t} U_{i 1}^{b *} U_{i 2}^{b} U_{j 2}^{t *} C_{00}^{b}, \\
& f_{26}^{v 2}=\frac{2 \sqrt{2} \alpha_{s} e g_{s} V_{t b}}{3 \pi \sin \theta_{W}} \sum_{i}^{2} \sum_{j}^{2} U_{j 1}^{t} U_{i 1}^{b *} U_{i 2}^{b} U_{j 2}^{t *} C_{12}^{b} \\
& f_{7}^{v 3}+f_{7}^{v 4}=-\frac{1}{2}\left(f_{15}^{v 3}+f_{15}^{v 4}\right)=\frac{\alpha_{s} e g_{s} V_{t b}}{12 \sqrt{2} \pi \sin \theta_{W}\left(t-m_{t}^{2}\right)} \sum_{i}^{2}\left[\left(18 C_{00}^{c}+2 C_{00}^{d}-9 B_{0}^{t}-9 m_{t}^{2} C_{1}^{e}\right) U_{i 1}^{t} U_{i 1}^{t *}\right. \\
& \left.-m_{t}\left(9 M_{\tilde{g}} U_{i 1}^{t} U_{i 2}^{t *} C_{0}^{c}+2 M_{\tilde{g}} U_{i 2}^{t} U_{i 1}^{t *} C_{0}^{c}+9 m_{t} U_{i 2}^{t} U_{i 2}^{t *} C_{1}^{e}\right)\right],
\end{aligned}
$$




$$
\begin{aligned}
& f_{9}^{v 3}+f_{9}^{v 4}=\frac{\alpha_{s} e g_{s} V_{t b}}{12 \sqrt{2} \pi \sin \theta_{W}\left(t-m_{t}^{2}\right)} \sum_{i}^{2}\left[\left(18 m_{t}^{2} C_{11}^{c}+18 m_{t}^{2} C_{12}^{c}+36 C_{00}^{c}-18 B_{0}^{t}-18 t C_{12}^{c}+4 C_{00}^{d}\right.\right. \\
& \left.+2 m_{t}^{2} C_{12}^{d}+2 m_{t}^{2} C_{11}^{d}-2 t C_{12}^{d}+m_{t}^{2} C_{1}^{d}\right) U_{i 1}^{t} U_{i 1}^{t *}+2 m_{t}^{2}\left(9 C_{11}^{c}+C_{1}^{d}+C_{11}^{d}\right) U_{i 2}^{t} U_{i 2}^{t *} \\
& \left.+2 m_{t} M_{\tilde{g}} U_{i 2}^{t} U_{i 1}^{t *}\left(9 C_{1}^{e}-C_{1}^{d}\right)+2 M_{\tilde{g}} m_{t} U_{i 1}^{t} U_{i 2}^{t *}\left(9 C_{1}^{e}-C_{1}^{d}\right)\right], \\
& f_{17}^{v 3}+f_{17}^{v 4}=\frac{\alpha_{s} e g_{s} V_{t b}}{12 \sqrt{2} \pi \sin \theta_{W}\left(t-m_{t}^{2}\right)} \sum_{i}^{2}\left[m_{t} U_{i 1}^{t} U_{i 1}^{t *}\left(9 B_{0}^{t}-18 C_{00}^{c}-2 C_{00}^{d}+9 m_{t}^{2} C_{1}^{e}-9 t C_{1}^{e}\right)\right. \\
& \left.+9 M_{\tilde{g}} U_{i 1}^{t} U_{i 2}^{t *}\left(m_{t}^{2}-t\right) C_{0}^{c}+m_{t} U_{i 2}^{t} U_{i 2}^{t *}\left(18 C_{00}^{c}-9 B_{0}^{t}+2 C_{00}^{d}\right)\right], \\
& f_{24}^{v 3}+f_{24}^{v 4}=-2\left(f_{37}^{v 3}+f_{37}^{v 4}\right)=\frac{\alpha_{s} e g_{s} V_{t b}}{3 \sqrt{2} \pi \sin \theta_{W}\left(t-m_{t}^{2}\right)} \sum_{i}^{2}\left[M_{\tilde{g}} U_{i 1}^{t} U_{i 2}^{t *}\left(C_{1}^{d}-9 C_{0}^{c}-9 C_{1}^{e}\right)\right. \\
& \left.+m_{t} U_{i 2}^{t} U_{i 2}^{t *}\left(9 C_{12}^{c}+C_{12}^{d}\right)-m_{t} U_{i 1}^{t} U_{i 1}^{t *}\left(9 C_{11}^{c}+9 C_{12}^{c}+C_{12}^{d}+C_{1}^{d}+C_{11}^{d}+9 C_{1}^{e}\right)\right], \\
& f_{1}^{v 5}+f_{1}^{v 6}=-2\left(f_{6}^{v 5}+f_{6}^{v 6}\right)=-\frac{3 \alpha_{s} e g_{s} V_{t b}}{2 \sqrt{2} \pi \sin \theta_{W}} \sum_{i}^{2} M_{\tilde{g}} U_{i 2}^{b} U_{i 1}^{b *} C_{0}^{f}, \\
& f_{7}^{v 5}+f_{7}^{v 6}=-\frac{1}{2}\left(f_{10}^{v 5}+f_{10}^{v 6}\right)=-\frac{\alpha_{s} e g_{s} V_{t b}}{12 \sqrt{2} \pi s \sin \theta_{W}} \sum_{i}^{2} U_{i 1}^{b} U_{i 1}^{b *}\left(9 B_{0}^{\prime s}-2 C_{00}^{g}-18 C_{00}^{f}\right), \\
& f_{8}^{v 5}+f_{8}^{v 6}=-\frac{\alpha_{s} e g_{s} V_{t b}}{6 \sqrt{2} \pi s \sin \theta_{W}} \sum_{i}^{2}\left[U_{i 1}^{b} U_{i 1}^{b *}\left(9 B_{0}^{\prime s}-2 C_{00}^{g}-18 C_{00}^{f}\right)+s U_{i 1}^{b} U_{i 1}^{b *}\left(9 C_{12}^{f}+C_{12}^{g}\right)\right], \\
& f_{38}^{v 5}+f_{10}^{v 6}=-\frac{\alpha_{s} e g_{s} V_{t b}}{6 \sqrt{2} \pi s \sin \theta_{W}} \sum_{i}^{2} M_{\tilde{g}} U_{i 2}^{b} U_{i 1}^{b *}\left(C_{1}^{g}-9 C_{1}^{f}-9 C_{0}^{f}\right), \\
& f_{1}^{v 7}=-\frac{\alpha_{s} e g_{s} V_{t b}}{6 \sqrt{2} \pi \sin \theta_{W}} \sum_{i}^{2} \sum_{j}^{2} U_{j 1}^{t} U_{i 1}^{b *} U_{i 2}^{b}\left(M_{\tilde{g}} U_{j 1}^{t *} C_{0}^{h}+m_{t} U_{j 2}^{t *} C_{2}^{h}\right), \\
& f_{22}^{v 7}=-\frac{\alpha_{s} e g_{s} V_{t b}}{6 \sqrt{2} \pi \sin \theta_{W}} \sum_{i}^{2} \sum_{j}^{2} U_{j 1}^{t} U_{i 1}^{b *} U_{i 1}^{b}\left(m_{t} U_{j 1}^{t *} C_{2}^{h}+M_{\tilde{g}} U_{j 2}^{t *} C_{0}^{h}\right) .
\end{aligned}
$$

As before, we define

$$
f_{m}^{b} \equiv \sum_{\beta=1}^{3} f_{m}^{b \beta}
$$

as shown in Fig 3, where

$$
\begin{gathered}
f_{1}^{b 1}=\frac{3 \alpha_{s} e g_{s} V_{t b}}{\sqrt{2} \pi \sin \theta_{W}} \sum_{i}^{2} \sum_{j}^{2} U_{j 1}^{t} U_{i 1}^{b *} U_{i 2}^{b}\left(M_{\tilde{g}} U_{j 1}^{t *} D_{00}^{a}+m_{t} U_{j 2}^{t *} D_{002}^{a}\right), \\
f_{2}^{b 1}=-\frac{3 \alpha_{s} e g_{s} V_{t b}}{\sqrt{2} \pi \sin \theta_{W}} \sum_{i}^{2} \sum_{j}^{2} U_{j 1}^{t} U_{i 1}^{b *} U_{i 2}^{b}\left(M_{\tilde{g}} U_{j 1}^{t *} D_{13}^{a}+m_{t} U_{j 2}^{t *} D_{123}^{a}\right),
\end{gathered}
$$




$$
\begin{aligned}
& f_{3}^{b 1}=\frac{3 \alpha_{s} e g_{s} V_{t b}}{\sqrt{2} \pi \sin \theta_{W}} \sum_{i}^{2} \sum_{j}^{2} U_{j 1}^{t} U_{i 1}^{b *}\left[M_{\tilde{g}} U_{i 2}^{b} U_{j 1}^{t *}\left(D_{1}^{a}+D_{11}^{a}+D_{12}^{a}\right)+m_{t} U_{i 2}^{b} U_{j 2}^{t *}\left(D_{112}^{a}+D_{12}^{a}+D_{122}^{a}\right)\right], \\
& f_{4}^{b 1}=\frac{3 \alpha_{s} e g_{s} V_{t b}}{\sqrt{2} \pi \sin \theta_{W}} \sum_{i}^{2} \sum_{j}^{2} U_{j 1}^{t} U_{i 1}^{b *}\left[M_{\tilde{g}} U_{i 2}^{b} U_{j 1}^{t *}\left(D_{12}^{a}+D_{2}^{a}+D_{22}^{a}\right)+m_{t} U_{i 2}^{b} U_{j 2}^{t *}\left(D_{122}^{a}+D_{22}^{a}+D_{222}^{a}\right)\right], \\
& f_{5}^{b 1}=-\frac{3 \alpha_{s} e g_{s} V_{t b}}{\sqrt{2} \pi \sin \theta_{W}} \sum_{i}^{2} \sum_{j}^{2} U_{j 1}^{t} U_{i 1}^{b *} U_{i 2}^{b}\left(M_{\tilde{g}} U_{j 1}^{t *} D_{23}^{a}+m_{t} U_{j 2}^{t *} D_{223}^{a}\right), \\
& f_{7}^{b 1}=-\frac{3 \alpha_{s} e g_{s} V_{t b}}{2 \sqrt{2} \pi \sin \theta_{W}} \sum_{i}^{2} \sum_{j}^{2} U_{j 1}^{t} U_{i 1}^{b *} U_{i 1}^{b} U_{j 1}^{t *} D_{00}^{a} \\
& f_{8}^{b 1}=\frac{3 \alpha_{s} e g_{s} V_{t b}}{\sqrt{2} \pi \sin \theta_{W}} \sum_{i}^{2} \sum_{j}^{2} U_{j 1}^{t} U_{i 1}^{b *} U_{i 1}^{b} U_{j 1}^{t *} D_{001}^{a} \\
& f_{9}^{b 1}=\frac{3 \alpha_{s} e g_{s} V_{t b}}{\sqrt{2} \pi \sin \theta_{W}} \sum_{i}^{2} \sum_{j}^{2} U_{j 1}^{t} U_{i 1}^{b *} U_{i 1}^{b} U_{j 1}^{t *} D_{002}^{a} \\
& f_{10}^{b 1}=-\frac{3 \alpha_{s} e g_{s} V_{t b}}{\sqrt{2} \pi \sin \theta_{W}} \sum_{i}^{2} \sum_{j}^{2} U_{j 1}^{t} U_{i 1}^{b *} U_{i 1}^{b} U_{j 1}^{t *}\left(D_{002}^{a}+D_{003}^{a}\right), \\
& f_{11}^{b 1}=-\frac{3 \alpha_{s} e g_{s} V_{t b}}{\sqrt{2} \pi \sin \theta_{W}} \sum_{i}^{2} \sum_{j}^{2} U_{j 1}^{t} U_{i 1}^{b *} U_{i 1}^{b} U_{j 1}^{t *}\left(D_{112}^{a}+D_{113}^{a}+D_{12}^{a}+D_{122}^{a}+D_{13}^{a}+D_{123}^{a}\right), \\
& f_{12}^{b 1}=-\frac{3 \alpha_{s} e g_{s} V_{t b}}{\sqrt{2} \pi \sin \theta_{W}} \sum_{i}^{2} \sum_{j}^{2} U_{j 1}^{t} U_{i 1}^{b *} U_{i 1}^{b} U_{j 1}^{t *}\left(D_{12}^{a}+D_{122}^{a}+D_{2}^{a}+2 D_{22}^{a}+D_{222}^{a}+D_{223}^{a}+D_{23}^{a}+D_{123}^{a}\right), \\
& f_{13}^{b 1}=\frac{3 \alpha_{s} e g_{s} V_{t b}}{\sqrt{2} \pi \sin \theta_{W}} \sum_{i}^{2} \sum_{j}^{2} U_{j 1}^{t} U_{i 1}^{b *} U_{i 1}^{b} U_{j 1}^{t *}\left(D_{133}^{a}+D_{123}^{a}\right), \\
& f_{14}^{b 1}=\frac{3 \alpha_{s} e g_{s} V_{t b}}{\sqrt{2} \pi \sin \theta_{W}} \sum_{i}^{2} \sum_{j}^{2} U_{j 1}^{t} U_{i 1}^{b *} U_{i 1}^{b} U_{j 1}^{t *}\left(D_{223}^{a}+D_{23}^{a}+D_{233}^{a}\right), \\
& f_{15}^{b 1}=-\frac{3 \alpha_{s} e g_{s} V_{t b}}{2 \sqrt{2} \pi \sin \theta_{W}} \sum_{i}^{2} \sum_{j}^{2} U_{j 1}^{t} U_{i 1}^{b *} U_{i 1}^{b} U_{j 1}^{t *}\left(2 D_{003}^{a}-C_{2}^{i}-2 D_{00}^{a}+m_{t}^{2} D_{23}^{a}-t D_{23}^{a}\right), \\
& f_{16}^{b 1}=-\frac{3 \alpha_{s} e g_{s} V_{t b}}{2 \sqrt{2} \pi \sin \theta_{W}} \sum_{i}^{2} \sum_{j}^{2} U_{j 1}^{t} U_{i 1}^{b *} U_{i 1}^{b} U_{j 1}^{t *}\left[\left(t-m_{t}^{2}\right)\left(D_{12}^{a}+D_{2}^{a}+D_{22}^{a}\right)-C_{2}^{i}-2\left(D_{00}^{a}+D_{001}^{a}+D_{002}^{a}\right)\right], \\
& f_{18}^{b 1}=-\frac{3 \alpha_{s} e g_{s} V_{t b}}{2 \sqrt{2} \pi \sin \theta_{W}} \sum_{i}^{2} \sum_{j}^{2} U_{j 1}^{t} U_{i 1}^{b *} M_{\tilde{g}} U_{i 2}^{b} U_{j 1}^{t *} D_{3}^{a}, \\
& f_{19}^{b 1}=\frac{3 \alpha_{s} e g_{s} V_{t b}}{2 \sqrt{2} \pi \sin \theta_{W}} \sum_{i}^{2} \sum_{j}^{2} U_{j 1}^{t} U_{i 1}^{b *}\left[M_{\tilde{g}} U_{i 2}^{b} U_{j 1}^{t *}\left(D_{0}^{a}+D_{1}^{a}+D_{2}^{a}\right)+m_{t} U_{i 2}^{b} U_{j 2}^{t *}\left(D_{12}^{a}+D_{2}^{a}+D_{22}^{a}-D_{23}^{a}\right)\right],
\end{aligned}
$$




$$
\begin{aligned}
& f_{20}^{b 1}=-\frac{3 \alpha_{s} e g_{s} V_{t b}}{\sqrt{2} \pi \sin \theta_{W}} \sum_{i}^{2} \sum_{j}^{2} U_{j 1}^{t} U_{i 1}^{b *} U_{i 1}^{b}\left(m_{t} U_{j 1}^{t *} D_{123}^{a}+M_{\tilde{g}} U_{j 2}^{t *} D_{13}^{a}\right), \\
& f_{21}^{b 1}=\frac{3 \alpha_{s} e g_{s} V_{t b}}{\sqrt{2} \pi \sin \theta_{W}} \sum_{i}^{2} \sum_{j}^{2} U_{j 1}^{t} U_{i 1}^{b *}\left[m_{t} U_{i 1}^{b} U_{j 1}^{t *}\left(D_{112}^{a}+D_{12}^{a}+D_{122}^{a}\right)+M_{\tilde{g}} U_{i 1}^{b} U_{j 2}^{t *}\left(D_{1}^{a}+D_{11}^{a}+D_{12}^{a}\right)\right] \text {, } \\
& f_{22}^{b 1}=\frac{3 \alpha_{s} e g_{s} V_{t b}}{\sqrt{2} \pi \sin \theta_{W}} \sum_{i}^{2} \sum_{j}^{2} U_{j 1}^{t} U_{i 1}^{b *} U_{i 1}^{b}\left(m_{t} U_{j 1}^{t *} D_{002}^{a}+M_{\tilde{g}} D_{00}^{a}\right), \\
& f_{23}^{b 1}=\frac{3 \alpha_{s} e g_{s} V_{t b}}{\sqrt{2} \pi \sin \theta_{W}} \sum_{i}^{2} \sum_{j}^{2} U_{j 1}^{t} U_{i 1}^{b *}\left[m_{t} U_{i 1}^{b} U_{j 1}^{t *}\left(D_{122}^{a}+D_{22}^{a}+D_{222}^{a}\right)+M_{\tilde{g}} U_{i 1}^{b} U_{j 2}^{t *}\left(D_{12}^{a}+D_{2}^{a}+D_{22}^{a}\right)\right] \text {, } \\
& f_{24}^{b 1}=-\frac{3 \alpha_{s} e g_{s} V_{t b}}{\sqrt{2} \pi \sin \theta_{W}} \sum_{i}^{2} \sum_{j}^{2} U_{j 1}^{t} U_{i 1}^{b *} U_{i 1}^{b}\left(m_{t} U_{j 1}^{t *} D_{223}^{a}+M_{\tilde{g}} U_{j 2}^{t *} D_{23}^{a}\right), \\
& f_{25}^{b 1}=-\frac{3 \alpha_{s} e g_{s} V_{t b}}{2 \sqrt{2} \pi \sin \theta_{W}} \sum_{i}^{2} \sum_{j}^{2} U_{j 1}^{t} U_{i 1}^{b *} U_{i 2}^{b} U_{j 2}^{t *}\left(m_{t}^{2} D_{23}^{a}-t D_{23}^{a}-A_{25}-2 D_{00}^{a}+2 D_{003}^{a}\right), \\
& f_{26}^{b 1}=-\frac{3 \alpha_{s} e g_{s} V_{t b}}{2 \sqrt{2} \pi \sin \theta_{W}} \sum_{i}^{2} \sum_{j}^{2} U_{j 1}^{t} U_{i 1}^{b *} U_{i 2}^{b} U_{j 2}^{t *}\left[\left(t-m_{t}^{2}\right)\left(D_{12}^{a}+D_{2}^{a}+D_{22}^{a}\right)-C_{2}^{i}-2\left(D_{00}^{a}+D_{001}^{a}+D_{002}^{a}\right)\right], \\
& f_{27}^{b 1}=-\frac{3 \alpha_{s} e g_{s} V_{t b}}{2 \sqrt{2} \pi \sin \theta_{W}} \sum_{i}^{2} \sum_{j}^{2} U_{j 1}^{t} U_{i 1}^{b *} U_{i 2}^{b} U_{j 2}^{t *} D_{00}^{a} \\
& f_{28}^{b 1}=-\frac{3 \alpha_{s} e g_{s} V_{t b}}{2 \sqrt{2} \pi \sin \theta_{W}} \sum_{i}^{2} \sum_{j}^{2} U_{j 1}^{t} U_{i 1}^{b *} U_{i 1}^{b}\left(m_{t} U_{j 1}^{t *} D_{23}^{a}+M_{\tilde{g}} U_{j 2}^{t *} D_{3}^{a}\right), \\
& f_{29}^{b 1}=-\frac{3 \alpha_{s} e g_{s} V_{t b}}{2 \sqrt{2} \pi \sin \theta_{W}} \sum_{i}^{2} \sum_{j}^{2} U_{j 1}^{t} U_{i 1}^{b *}\left[m_{t} U_{i 1}^{b} U_{j 1}^{t *}\left(D_{12}^{a}+D_{2}^{a}+D_{22}^{a}\right)+M_{\tilde{g}} U_{i 1}^{b} U_{j 2}^{t *}\left(D_{0}^{a}+D_{1}^{a}+D_{2}^{a}\right)\right] \text {, } \\
& f_{30}^{b 1}=\frac{3 \alpha_{s} e g_{s} V_{t b}}{\sqrt{2} \pi \sin \theta_{W}} \sum_{i}^{2} \sum_{j}^{2} U_{j 1}^{t} U_{i 1}^{b *} U_{i 2}^{b} U_{j 2}^{t *} D_{001}^{a} \\
& f_{31}^{b 1}=\frac{3 \alpha_{s} e g_{s} V_{t b}}{\sqrt{2} \pi \sin \theta_{W}} \sum_{i}^{2} \sum_{j}^{2} U_{j 1}^{t} U_{i 1}^{b *} U_{i 2}^{b} U_{j 2}^{t *} D_{002}^{a} \\
& f_{32}^{b 1}=-\frac{3 \alpha_{s} e g_{s} V_{t b}}{\sqrt{2} \pi \sin \theta_{W}} \sum_{i}^{2} \sum_{j}^{2} U_{j 1}^{t} U_{i 1}^{b *} U_{i 2}^{b} U_{j 2}^{t *}\left(D_{002}^{a}+D_{003}^{a}\right), \\
& f_{33}^{b 1}=-\frac{3 \alpha_{s} e g_{s} V_{t b}}{\sqrt{2} \pi \sin \theta_{W}} \sum_{i}^{2} \sum_{j}^{2} U_{j 1}^{t} U_{i 1}^{b *} U_{i 2}^{b} U_{j 2}^{t *}\left(D_{112}^{a}+D_{113}^{a}+D_{12}^{a}+D_{122}^{a}+D_{13}^{a}+D_{123}^{a}\right), \\
& f_{34}^{b 1}=-\frac{3 \alpha_{s} e g_{s} V_{t b}}{\sqrt{2} \pi \sin \theta_{W}} \sum_{i}^{2} \sum_{j}^{2} U_{j 1}^{t} U_{i 1}^{b *} U_{i 2}^{b} U_{j 2}^{t *}\left(D_{12}^{a}+D_{122}^{a}+D_{2}^{a}+2 D_{22}^{a}+D_{222}^{a}+D_{223}^{a}+D_{23}^{a}+D_{123}^{a}\right),
\end{aligned}
$$




$$
\begin{aligned}
& f_{35}^{b 1}=\frac{3 \alpha_{s} e g_{s} V_{t b}}{\sqrt{2} \pi \sin \theta_{W}} \sum_{i}^{2} \sum_{j}^{2} U_{j 1}^{t} U_{i 1}^{b *} U_{i 2}^{b} U_{j 2}^{t *}\left(D_{133}^{a}+D_{123}^{a}\right) \\
& f_{36}^{b 1}=\frac{3 \alpha_{s} e g_{s} V_{t b}}{\sqrt{2} \pi \sin \theta_{W}} \sum_{i}^{2} \sum_{j}^{2} U_{j 1}^{t} U_{i 1}^{b *} U_{i 2}^{b} U_{j 2}^{t *}\left(D_{223}^{a}+D_{23}^{a}+D_{233}^{a}\right), \\
& f_{1}^{b 2}=\frac{\alpha_{s} e g_{s} V_{t b}}{3 \sqrt{2} \pi \sin \theta_{W}} \sum_{i}^{2} \sum_{j}^{2} U_{j 1}^{t} U_{i 1}^{b *}\left(M_{\tilde{g}} U_{i 2}^{b} U_{j 1}^{t *} D_{00}^{b}+m_{t} U_{i 2}^{b} U_{j 2}^{t *} D_{003}^{b}\right), \\
& f_{2}^{b 2}=-\frac{\alpha_{s} e g_{s} V_{t b}}{3 \sqrt{2} \pi \sin \theta_{W}} \sum_{i}^{2} \sum_{j}^{2} U_{j 1}^{t} U_{i 1}^{b *}\left[M_{\tilde{g}} U_{i 2}^{b} U_{j 1}^{t *}\left(D_{1}^{b}+D_{12}^{b}+D_{23}^{b}+D_{3}^{b}+D_{33}^{b}+D_{13}^{b}\right)\right. \\
& \left.+m_{t} U_{i 2}^{b} U_{j 2}^{t *}\left(D_{123}^{b}+D_{13}^{b}+D_{233}^{b}+D_{33}^{b}+D_{333}^{b}+D_{133}^{b}\right)\right], \\
& f_{3}^{b 2}=\frac{\alpha_{s} e g_{s} V_{t b}}{3 \sqrt{2} \pi \sin \theta_{W}} \sum_{i}^{2} \sum_{j}^{2} U_{j 1}^{t} U_{i 1}^{b *}\left[M_{\tilde{g}} U_{i 2}^{b} U_{j 1}^{t *}\left(D_{11}^{b}+D_{13}^{b}\right)+m_{t} U_{i 2}^{b} U_{j 2}^{t *}\left(D_{113}^{b}+D_{133}^{b}\right)\right] \\
& f_{4}^{b 2}=-\frac{\alpha_{s} e g_{s} V_{t b}}{3 \sqrt{2} \pi \sin \theta_{W}} \sum_{i}^{2} \sum_{j}^{2} U_{j 1}^{t} U_{i 1}^{b *}\left(M_{\tilde{g}} U_{i 2}^{b} U_{j 1}^{t *} D_{13}^{b}+m_{t} U_{i 2}^{b} U_{j 2}^{t *} D_{133}^{b}\right) \\
& f_{5}^{b 2}=\frac{\alpha_{s} e g_{s} V_{t b}}{3 \sqrt{2} \pi \sin \theta_{W}} \sum_{i}^{2} \sum_{j}^{2} U_{j 1}^{t} U_{i 1}^{b *}\left[M_{\tilde{g}} U_{i 2}^{b} U_{j 1}^{t *}\left(D_{23}^{b}+D_{3}^{b}+D_{33}^{b}\right)+m_{t} U_{i 2}^{b} U_{j 2}^{t *}\left(D_{233}^{b}+D_{33}^{b}+D_{333}^{b}\right)\right], \\
& f_{8}^{b 2}=-\frac{\alpha_{s} e g_{s} V_{t b}}{3 \sqrt{2} \pi \sin \theta_{W}} \sum_{i}^{2} \sum_{j}^{2} U_{j 1}^{t} U_{i 1}^{b *} U_{i 1}^{b} U_{j 1}^{t *}\left(D_{001}^{b}+D_{003}^{b}\right) \\
& f_{9}^{b 2}=\frac{\alpha_{s} e g_{s} V_{t b}}{3 \sqrt{2} \pi \sin \theta_{W}} \sum_{i}^{2} \sum_{j}^{2} U_{j 1}^{t} U_{i 1}^{b *} U_{i 1}^{b} U_{j 1}^{t *} D_{003}^{b} \\
& f_{10}^{b 2}=\frac{\alpha_{s} e g_{s} V_{t b}}{3 \sqrt{2} \pi \sin \theta_{W}} \sum_{i}^{2} \sum_{j}^{2} U_{j 1}^{t} U_{i 1}^{b *} U_{i 1}^{b} U_{j 1}^{t *} D_{002}^{b} \\
& f_{11}^{b 2}=\frac{\alpha_{s} e g_{s} V_{t b}}{3 \sqrt{2} \pi \sin \theta_{W}} \sum_{i}^{2} \sum_{j}^{2} U_{j 1}^{t} U_{i 1}^{b *} U_{i 1}^{b} U_{j 1}^{t *}\left(D_{112}^{b}+D_{123}^{b}\right) \\
& f_{12}^{b 2}=-\frac{\alpha_{s} e g_{s} V_{t b}}{3 \sqrt{2} \pi \sin \theta_{W}} \sum_{i}^{2} \sum_{j}^{2} U_{j 1}^{t} U_{i 1}^{b *} U_{i 1}^{b} U_{j 1}^{t *} D_{123}^{b}, \\
& f_{13}^{b 2}=-\frac{\alpha_{s} e g_{s} V_{t b}}{3 \sqrt{2} \pi \sin \theta_{W}} \sum_{i}^{2} \sum_{j}^{2} U_{j 1}^{t} U_{i 1}^{b *} U_{i 1}^{b} U_{j 1}^{t *}\left(D_{223}^{b}+D_{23}^{b}+D_{233}^{b}+D_{12}^{b}+D_{122}^{b}+D_{123}^{b}\right), \\
& f_{14}^{b 2}=\frac{\alpha_{s} e g_{s} V_{t b}}{3 \sqrt{2} \pi \sin \theta_{W}} \sum_{i}^{2} \sum_{j}^{2} U_{j 1}^{t} U_{i 1}^{b *} U_{i 1}^{b} U_{j 1}^{t *}\left(D_{223}^{b}+D_{23}^{b}+D_{233}^{b}\right),
\end{aligned}
$$




$$
\begin{aligned}
& f_{15}^{b 2}=\frac{\alpha_{s} e g_{s} V_{t b}}{3 \sqrt{2} \pi \sin \theta_{W}} \sum_{i}^{2} \sum_{j}^{2} U_{j 1}^{t} U_{i 1}^{b *} U_{i 1}^{b} U_{j 1}^{t *}\left(D_{00}^{b}+D_{002}^{b}+D_{003}^{b}\right), \\
& f_{16}^{b 2}=-\frac{\alpha_{s} e g_{s} V_{t b}}{3 \sqrt{2} \pi \sin \theta_{W}} \sum_{i}^{2} \sum_{j}^{2} U_{j 1}^{t} U_{i 1}^{b *} U_{i 1}^{b} U_{j 1}^{t *} D_{001}^{b}, \\
& f_{20}^{b 2}=-\frac{\alpha_{s} e g_{s} V_{t b}}{3 \sqrt{2} \pi \sin \theta_{W}} \sum_{i}^{2} \sum_{j}^{2} U_{j 1}^{t} U_{i 1}^{b *}\left[m_{t} U_{i 1}^{b} U_{j 1}^{t *}\left(D_{123}^{b}+D_{13}^{b}+D_{233}^{b}+D_{33}^{b}+D_{333}^{b}+D_{133}^{b}\right)\right. \\
& \left.+M_{\tilde{g}} U_{i 1}^{b} U_{j 2}^{t *}\left(D_{1}^{b}+D_{12}^{b}+D_{23}^{b}+D_{3}^{b}+D_{33}^{b}+D_{13}^{b}\right)\right], \\
& f_{21}^{b 2}=\frac{\alpha_{s} e g_{s} V_{t b}}{3 \sqrt{2} \pi \sin \theta_{W}} \sum_{i}^{2} \sum_{j}^{2} U_{j 1}^{t} U_{i 1}^{b *}\left[m_{t} U_{i 1}^{b} U_{j 1}^{t *}\left(D_{113}^{b}+D_{133}^{b}\right)+M_{\tilde{g}} U_{i 1}^{b} U_{j 2}^{t *}\left(D_{11}^{b}+D_{13}^{b}\right)\right], \\
& f_{22}^{b 2}=\frac{\alpha_{s} e g_{s} V_{t b}}{3 \sqrt{2} \pi \sin \theta_{W}} \sum_{i}^{2} \sum_{j}^{2} U_{j 1}^{t} U_{i 1}^{b *}\left(m_{t} U_{i 1}^{b} U_{j 1}^{t *} D_{003}^{b}+M_{\tilde{g}} U_{i 1}^{b} U_{j 2}^{t *} D_{00}^{b}\right) \\
& f_{23}^{b 2}=-\frac{\alpha_{s} e g_{s} V_{t b}}{3 \sqrt{2} \pi \sin \theta_{W}} \sum_{i}^{2} \sum_{j}^{2} U_{j 1}^{t} U_{i 1}^{b *}\left(m_{t} U_{i 1}^{b} U_{j 1}^{t *} D_{133}^{b}+M_{\tilde{g}} U_{i 1}^{b} U_{j 2}^{t *} D_{13}^{b}\right) \\
& f_{24}^{b 2}=\frac{\alpha_{s} e g_{s} V_{t b}}{3 \sqrt{2} \pi \sin \theta_{W}} \sum_{i}^{2} \sum_{j}^{2} U_{j 1}^{t} U_{i 1}^{b *}\left[M_{\tilde{g}} U_{i 1}^{b} U_{j 2}^{t *}\left(D_{23}^{b}+D_{3}^{b}+D_{33}^{b}\right)+m_{t} U_{i 1}^{b} U_{j 1}^{t *}\left(D_{233}^{b}+D_{33}^{b}+D_{333}^{b}\right)\right], \\
& f_{25}^{b 2}=\frac{\alpha_{s} e g_{s} V_{t b}}{3 \sqrt{2} \pi \sin \theta_{W}} \sum_{i}^{2} \sum_{j}^{2} U_{j 1}^{t} U_{i 1}^{b *} U_{i 2}^{b} U_{j 2}^{t *}\left(D_{00}^{b}+D_{002}^{b}+D_{003}^{b}\right), \\
& f_{26}^{b 2}=-\frac{\alpha_{s} e g_{s} V_{t b}}{3 \sqrt{2} \pi \sin \theta_{W}} \sum_{i}^{2} \sum_{j}^{2} U_{j 1}^{t} U_{i 1}^{b *} U_{i 2}^{b} U_{j 2}^{t *} D_{001}^{b} \\
& f_{30}^{b 2}=-\frac{\alpha_{s} e g_{s} V_{t b}}{3 \sqrt{2} \pi \sin \theta_{W}} \sum_{i}^{2} \sum_{j}^{2} U_{j 1}^{t} U_{i 1}^{b *} U_{i 2}^{b} U_{j 2}^{t *}\left(D_{001}^{b}+D_{003}^{b}\right), \\
& f_{31}^{b 2}=\frac{\alpha_{s} e g_{s} V_{t b}}{3 \sqrt{2} \pi \sin \theta_{W}} \sum_{i}^{2} \sum_{j}^{2} U_{j 1}^{t} U_{i 1}^{b *} U_{i 2}^{b} U_{j 2}^{t *} D_{003}^{b}, \\
& f_{32}^{b 2}=\frac{\alpha_{s} e g_{s} V_{t b}}{3 \sqrt{2} \pi \sin \theta_{W}} \sum_{i}^{2} \sum_{j}^{2} U_{j 1}^{t} U_{i 1}^{b *} U_{i 2}^{b} U_{j 2}^{t *} D_{002}^{b} \\
& f_{33}^{b 2}=\frac{\alpha_{s} e g_{s} V_{t b}}{3 \sqrt{2} \pi \sin \theta_{W}} \sum_{i}^{2} \sum_{j}^{2} U_{j 1}^{t} U_{i 1}^{b *} U_{i 2}^{b} U_{j 2}^{t *}\left(D_{112}^{b}+D_{123}^{b}\right), \\
& f_{34}^{b 2}=-\frac{\alpha_{s} e g_{s} V_{t b}}{3 \sqrt{2} \pi \sin \theta_{W}} \sum_{i}^{2} \sum_{j}^{2} U_{j 1}^{t} U_{i 1}^{b *} U_{i 2}^{b} U_{j 2}^{t *} D_{123}^{b},
\end{aligned}
$$




$$
\begin{aligned}
& f_{35}^{b 2}=-\frac{\alpha_{s} e g_{s} V_{t b}}{3 \sqrt{2} \pi \sin \theta_{W}} \sum_{i}^{2} \sum_{j}^{2} U_{j 1}^{t} U_{i 1}^{b *} U_{i 2}^{b} U_{j 2}^{t *}\left(D_{12}^{b}+D_{122}^{b}+D_{223}^{b}+D_{23}^{b}+D_{233}^{b}+D_{123}^{b}\right), \\
& f_{36}^{b 2}=\frac{\alpha_{s} e g_{s} V_{t b}}{3 \sqrt{2} \pi \sin \theta_{W}} \sum_{i}^{2} \sum_{j}^{2} U_{j 1}^{t} U_{i 1}^{b *} U_{i 2}^{b} U_{j 2}^{t *}\left(D_{223}^{b}+D_{23}^{b}+D_{233}^{b}\right), \\
& f_{1}^{b 3}=-\frac{\alpha_{s} e g_{s} V_{t b}}{3 \sqrt{2} \pi \sin \theta_{W}} \sum_{i}^{2} \sum_{j}^{2} U_{i 1}^{t} U_{j 1}^{b *}\left[m_{t} U_{j 2}^{b} U_{i 2}^{t *}\left(D_{00}^{c}+D_{001}^{c}+D_{003}^{c}\right)-M_{\tilde{g}} U_{j 2}^{b} U_{i 1}^{t *} D_{00}^{c}\right] \\
& f_{2}^{b 3}=-\frac{\alpha_{s} e g_{s} V_{t b}}{3 \sqrt{2} \pi \sin \theta_{W}} \sum_{i}^{2} \sum_{j}^{2} U_{i 1}^{t} U_{j 1}^{b *}\left[M_{\tilde{g}} U_{j 2}^{b} U_{i 1}^{t *}\left(D_{23}^{c}+D_{13}^{c}+D_{3}^{c}+D_{33}^{c}\right)\right. \\
& \left.-m_{t} U_{j 2}^{b} U_{i 2}^{t *}\left(D_{113}^{c}+2 D_{133}^{c}+D_{123}^{c}+2 D_{13}^{c}+D_{23}^{c}+D_{233}^{c}+D_{3}^{c}+2 D_{33}^{c}+D_{333}^{c}\right)\right] \text {, } \\
& f_{3}^{b 3}=-\frac{\alpha_{s} e g_{s} V_{t b}}{3 \sqrt{2} \pi \sin \theta_{W}} \sum_{i}^{2} \sum_{j}^{2} U_{i 1}^{t} U_{j 1}^{b *}\left[M_{\tilde{g}} U_{j 2}^{b} U_{i 1}^{t *} D_{13}^{c}-m_{t} U_{j 2}^{b} U_{i 2}^{t *}\left(D_{113}^{c}+D_{133}^{c}+D_{13}^{c}\right)\right], \\
& f_{4}^{b 3}=-\frac{\alpha_{s} e g_{s} V_{t b}}{3 \sqrt{2} \pi \sin \theta_{W}} \sum_{i}^{2} \sum_{j}^{2} U_{i 1}^{t} U_{j 1}^{b *}\left[m_{t} U_{j 2}^{b} U_{i 2}^{t *}\left(D_{11}^{c}+D_{111}^{c}+2 D_{113}^{c}+D_{133}^{c}+D_{13}^{c}\right)\right. \\
& \left.-M_{\tilde{g}} U_{j 2}^{b} U_{i 1}^{t *}\left(D_{11}^{c}+D_{13}^{c}\right)\right] \\
& f_{5}^{b 3}=-\frac{\alpha_{s} e g_{s} V_{t b}}{3 \sqrt{2} \pi \sin \theta_{W}} \sum_{i}^{2} \sum_{j}^{2} U_{i 1}^{t} U_{j 1}^{b *}\left[m _ { t } U _ { j 2 } ^ { b } U _ { i 2 } ^ { t * } \left(2 D_{11}^{c}+D_{111}^{c}+3 D_{113}^{c}+3 D_{133}^{c}+2 D_{123}^{c}+4 D_{13}^{c}\right.\right. \\
& \left.+D_{23}^{c}+D_{233}^{c}+D_{3}^{c}+2 D_{33}^{c}+D_{333}^{c}+D_{1}^{c}+D_{112}^{c}+D_{12}^{c}\right) \\
& \left.-M_{\tilde{g}} U_{j 2}^{b} U_{i 1}^{t *}\left(D_{1}^{c}+D_{11}^{c}+D_{12}^{c}+D_{23}^{c}+2 D_{13}^{c}+D_{3}^{c}+D_{33}^{c}\right)\right], \\
& f_{8}^{b 3}=\frac{\alpha_{s} e g_{s} V_{t b}}{3 \sqrt{2} \pi \sin \theta_{W}} \sum_{i}^{2} \sum_{j}^{2} U_{i 1}^{t} U_{j 1}^{b *} U_{j 1}^{b} U_{i 1}^{t *} D_{003}^{c}, \\
& f_{9}^{b 3}=-\frac{\alpha_{s} e g_{s} V_{t b}}{3 \sqrt{2} \pi \sin \theta_{W}} \sum_{i}^{2} \sum_{j}^{2} U_{i 1}^{t} U_{j 1}^{b *} U_{j 1}^{b} U_{i 1}^{t *}\left(D_{001}^{c}-D_{003}^{c}\right), \\
& f_{10}^{b 3}=-\frac{\alpha_{s} e g_{s} V_{t b}}{3 \sqrt{2} \pi \sin \theta_{W}} \sum_{i}^{2} \sum_{j}^{2} U_{i 1}^{t} U_{j 1}^{b *} U_{j 1}^{b} U_{i 1}^{t *} D_{002}^{c}, \\
& f_{11}^{b 3}=\frac{\alpha_{s} e g_{s} V_{t b}}{3 \sqrt{2} \pi \sin \theta_{W}} \sum_{i}^{2} \sum_{j}^{2} U_{i 1}^{t} U_{j 1}^{b *} U_{j 1}^{b} U_{i 1}^{t *} D_{123}^{c}, \\
& f_{12}^{b 3}=-\frac{\alpha_{s} e g_{s} V_{t b}}{3 \sqrt{2} \pi \sin \theta_{W}} \sum_{i}^{2} \sum_{j}^{2} U_{i 1}^{t} U_{j 1}^{b *} U_{j 1}^{b} U_{i 1}^{t *}\left(D_{112}^{c}+D_{123}^{c}\right), \\
& f_{13}^{b 3}=\frac{\alpha_{s} e g_{s} V_{t b}}{3 \sqrt{2} \pi \sin \theta_{W}} \sum_{i}^{2} \sum_{j}^{2} U_{i 1}^{t} U_{j 1}^{b *} U_{j 1}^{b} U_{i 1}^{t *}\left(D_{123}^{c}+D_{223}^{c}+D_{23}^{c}+D_{233}^{c}\right),
\end{aligned}
$$




$$
\begin{aligned}
& f_{14}^{b 3}=-\frac{\alpha_{s} e g_{s} V_{t b}}{3 \sqrt{2} \pi \sin \theta_{W}} \sum_{i}^{2} \sum_{j}^{2} U_{i 1}^{t} U_{j 1}^{b *} U_{j 1}^{b} U_{i 1}^{t *}\left(D_{112}^{c}+D_{12}^{c}+D_{122}^{c}+2 D_{123}^{c}+D_{223}^{c}+D_{23}^{c}+D_{233}^{c}\right), \\
& f_{15}^{b 3}=-\frac{\alpha_{s} e g_{s} V_{t b}}{3 \sqrt{2} \pi \sin \theta_{W}} \sum_{i}^{2} \sum_{j}^{2} U_{i 1}^{t} U_{j 1}^{b *} U_{j 1}^{b} U_{i 1}^{t *}\left(D_{00}^{c}+2 D_{001}^{c}+D_{002}^{c}+D_{003}^{c}\right), \\
& f_{20}^{b 3}=-\frac{\alpha_{s} e g_{s} V_{t b}}{3 \sqrt{2} \pi \sin \theta_{W}} \sum_{i}^{2} \sum_{j}^{2} U_{i 1}^{t} U_{j 1}^{b *}\left[M_{\tilde{g}} U_{j 1}^{b} U_{i 2}^{t *}\left(D_{13}^{c}+D_{23}^{c}+D_{3}^{c}+D_{33}^{c}\right)\right. \\
& \left.-m_{t} U_{j 1}^{b} U_{i 1}^{t *}\left(D_{113}^{c}+2 D_{13}^{c}+D_{123}^{c}+2 D_{133}^{c}+D_{23}^{c}+D_{233}^{c}+D_{3}^{c}+2 D_{33}^{c}+D_{333}^{c}\right)\right] \text {, } \\
& f_{21}^{b 3}=-\frac{\alpha_{s} e g_{s} V_{t b}}{3 \sqrt{2} \pi \sin \theta_{W}} \sum_{i}^{2} \sum_{j}^{2} U_{i 1}^{t} U_{j 1}^{b *}\left[M_{\tilde{g}} U_{j 1}^{b} U_{i 2}^{t *} D_{13}^{c}-m_{t} U_{j 1}^{b} U_{i 1}^{t *}\left(D_{113}^{c}+D_{13}^{c}+D_{133}^{c}\right)\right], \\
& f_{22}^{b 3}=-\frac{\alpha_{s} e g_{s} V_{t b}}{3 \sqrt{2} \pi \sin \theta_{W}} \sum_{i}^{2} \sum_{j}^{2} U_{i 1}^{t} U_{j 1}^{b *}\left[m_{t} U_{j 1}^{b} U_{i 1}^{t *}\left(D_{00}^{c}+D_{001}^{c}+D_{003}^{c}\right)-M_{\tilde{g}} U_{j 1}^{b} U_{i 2}^{t *} D_{00}^{c}\right], \\
& f_{23}^{b 3}=-\frac{\alpha_{s} e g_{s} V_{t b}}{3 \sqrt{2} \pi \sin \theta_{W}} \sum_{i}^{2} \sum_{j}^{2} U_{i 1}^{t} U_{j 1}^{b *}\left[m_{t} U_{j 1}^{b} U_{i 1}^{t *}\left(D_{11}^{c}+D_{111}^{c}+2 D_{113}^{c}+D_{13}^{c}+D_{133}^{c}\right)\right. \\
& \left.-M_{\tilde{g}} U_{j 1}^{b} U_{i 2}^{t *}\left(D_{11}^{c}+D_{13}^{c}\right)\right] \\
& f_{24}^{b 3}=-\frac{\alpha_{s} e g_{s} V_{t b}}{3 \sqrt{2} \pi \sin \theta_{W}} \sum_{i}^{2} \sum_{j}^{2} U_{i 1}^{t} U_{j 1}^{b *}\left[m _ { t } U _ { j 1 } ^ { b } U _ { i 1 } ^ { t * } \left(D_{1}^{c}+2 D_{11}^{c}+D_{112}^{c}+D_{111}^{c}+3 D_{113}^{c}+D_{12}^{c}+4 D_{13}^{c}\right.\right. \\
& \left.+2 D_{123}^{c}+3 D_{133}^{c}+D_{23}^{c}+D_{233}^{c}+D_{3}^{c}+2 D_{33}^{c}+D_{333}^{c}\right) \\
& \left.-M_{\tilde{g}} U_{j 1}^{b} U_{i 2}^{t *}\left(D_{11}^{c}+D_{12}^{c}+D_{1}^{c}+2 D_{13}^{c}+D_{23}^{c}+D_{3}^{c}+D_{33}^{c}\right)\right], \\
& f_{25}^{b 3}=-\frac{\alpha_{s} e g_{s} V_{t b}}{3 \sqrt{2} \pi \sin \theta_{W}} \sum_{i}^{2} \sum_{j}^{2} U_{i 1}^{t} U_{j 1}^{b *} U_{j 2}^{b} U_{i 2}^{t *}\left(D_{00}^{c}+D_{002}^{c}+D_{003}^{c}+D_{001}^{c}\right), \\
& f_{26}^{b 3}=-\frac{\alpha_{s} e g_{s} V_{t b}}{3 \sqrt{2} \pi \sin \theta_{W}} \sum_{i}^{2} \sum_{j}^{2} U_{i 1}^{t} U_{j 1}^{b *} U_{j 2}^{b} U_{i 2}^{t *} D_{001}^{c}, \\
& f_{30}^{b 3}=\frac{\alpha_{s} e g_{s} V_{t b}}{3 \sqrt{2} \pi \sin \theta_{W}} \sum_{i}^{2} \sum_{j}^{2} U_{i 1}^{t} U_{j 1}^{b *} U_{j 2}^{b} U_{i 2}^{t *} D_{003}^{c}, \\
& f_{31}^{b 3}=-\frac{\alpha_{s} e g_{s} V_{t b}}{3 \sqrt{2} \pi \sin \theta_{W}} \sum_{i}^{2} \sum_{j}^{2} U_{i 1}^{t} U_{j 1}^{b *} U_{j 2}^{b} U_{i 2}^{t *}\left(D_{001}^{c}+D_{003}^{c}\right), \\
& f_{32}^{b 3}=-\frac{\alpha_{s} e g_{s} V_{t b}}{3 \sqrt{2} \pi \sin \theta_{W}} \sum_{i}^{2} \sum_{j}^{2} U_{i 1}^{t} U_{j 1}^{b *} U_{j 2}^{b} U_{i 2}^{t *} D_{002}^{c}, \\
& f_{33}^{b 3}=\frac{\alpha_{s} e g_{s} V_{t b}}{3 \sqrt{2} \pi \sin \theta_{W}} \sum_{i}^{2} \sum_{j}^{2} U_{i 1}^{t} U_{j 1}^{b *} U_{j 2}^{b} U_{i 2}^{t *} D_{123}^{c},
\end{aligned}
$$




$$
\begin{gathered}
f_{34}^{b 3}=-\frac{\alpha_{s} e g_{s} V_{t b}}{3 \sqrt{2} \pi \sin \theta_{W}} \sum_{i}^{2} \sum_{j}^{2} U_{i 1}^{t} U_{j 1}^{b *} U_{j 2}^{b} U_{i 2}^{t *}\left(D_{112}^{c}+D_{123}^{c}\right), \\
f_{35}^{b 3}=\frac{\alpha_{s} e g_{s} V_{t b}}{3 \sqrt{2} \pi \sin \theta_{W}} \sum_{i}^{2} \sum_{j}^{2} U_{i 1}^{t} U_{j 1}^{b *} U_{j 2}^{b} U_{i 2}^{t *}\left(D_{223}^{c}+D_{23}^{c}+D_{233}^{c}+D_{123}^{c}\right), \\
f_{36}^{b 3}=-\frac{\alpha_{s} e g_{s} V_{t b}}{3 \sqrt{2} \pi \sin \theta_{W}} \sum_{i}^{2} \sum_{j}^{2} U_{i 1}^{t} U_{j 1}^{b *} U_{j 2}^{b} U_{i 2}^{t *}\left(D_{223}^{c}+D_{23}^{c}+D_{233}^{c}+D_{112}^{c}+D_{12}^{c}+D_{122}^{c}+2 D_{123}^{c}\right) .
\end{gathered}
$$


[1] the Report of the "1999 CERN Workshop on SM physics (and more) at the LHC", hep-ph/0003033.

[2] Wolfgang Wagner, Rep.Prog.Phys. 68, (2005).

[3] A.P.Heinson, A.S.Belyaev and E.E.Boos, Phys. Rev. D56, 3114 (1997) hep-ph/9612424.

[4] D.O.Carlson and C.P.Yuan, Phys. Lett. B306, 386(1993).

[5] G.Mahlon and S.Parke, Phys. Rev. D55, 7249 (1997) hep-ph/9611367.

[6] G.Mahlon and S.Parke, hep-ph/9912458.

[7] D.O.Carlson, E.Malkawi and C.P.Yuan, Phys. Lett. B337,145 (1994) hep-ph/9405277; A.Datta and X.Zhang, Phys. Rev. D55, 2530 (1997) hep-ph/9611247.

[8] T.Tait and C.P.Yuan, hep-ph/9710372.

[9] K.Hikasa, K.Whisnant, J.M.Yang and B.Young, Phys. Rev. D58, 114003 (1998) hep-ph/9806401.

[10] E.Boos, L.Dudko and T.Ohl, Eur. Phys. J. C11, 473 (1999) hep-ph/9903215.

[11] T. Han, M. Hosch, K. Whisnant, B. Young and X. Zhang, Phys. Rev. D58, 073008 (1998) hep-ph/9806486.

[12] J. J. Liu, C. S. Li, L. L. Yang and L. G. Jin, Nucl. Phys. B 705, 3 (2005) hep-ph/0404099.

[13] J. J. Liu, C. S. Li, L. L. Yang and L. G. Jin, Mod. Phys. Lett. A 19, 317 (2004).

[14] D.Atwood, S.Bar-Shalom, G.Eilam and A.Soni, Phys. Rev. D54, 5412 (1996) hep-ph/9605345.

[15] E.H.Simmons, Phys. Rev. D55, 5494 (1997) hep-ph/9612402.

[16] C.S.Li, R.J.Oakes and J.M.Yang, Phys. Rev. D55, 1672 (1997) hep-ph/9608460.

[17] C.S.Li, R.J.Oakes and J.M.Yang, Phys. Rev. D55, 5780 (1997) hep-ph/9611455; C.S.Li, R.J.Oakes, J.M.Yang and H.Y.Zhou, Phys. Rev. D57, 2009(1998) hep-ph/9706412.

[18] S.Bar-Shalom, D.Atwood and A.Soni, Phys. Rev. D57,1495 (1998) hep-ph/9708357.

[19] S.S.Willenbrock and D.A.Dicus, Phys. Rev. D34, 155 (1986); C.P.Yuan, Phys. Rev. D41, 42 (1990); R.K.Ellis and S.Parke, Phys. Rev. D46, 3785 (1992).

[20] S.Cortese and R.Petronzio, Phys. Lett. B253, 494 (1991).

[21] T.Stelzer and S.Willenbrock, Phys. Lett. B357, 125 (1995) hep-ph/9505433.

[22] T.M.Tait, Phys. Rev. D61,034001 (2000) hep-ph/9909352.

[23] D.Green et al., " A Study of Single Top at CMS," CMS Note 1999/048, unpublished. 
[24] B. González Piñeiro, D.O’Neil, R.Brock and M.Lefebvre, "Measuring $V_{t b}$ and Polarization via Boson Gluon Fusion at the LHC", ATLAS Note: ATL-COM-PHYS-99-027, unpublished.

[25] ATLAS Collab., "ATLAS Detector and Physics Performance Technical Design Report", CERN LHCC 99-14/15 (1999).

[26] T.Stelzer, Z.Sullivan and S.Willenbrock, Phys. Rev. D56, 5919 (1997) hep-ph/9705398.

[27] M.C.Smith and S.Willenbrock, Phys. Rev. D54, 6696 (1996) hep-ph/9604223.

[28] Shouhua Zhu, KA-TP-27-2001, hep-ph/0109269.

[29] N. Kidonakis, hep-ph/0609287.

[30] M. Beccaria, G. Macorini, F. M. Renard and C. Verzegnassi, hep-ph/0605108.

[31] M. Beccaria, G. Macorini, F. M. Renard and C. Verzegnassi, "Associated t W production at LHC: A complete calculation of electroweak supersymmetric effects at one loop," Phys. Rev. D 73, 093001 (2006) arXiv:hep-ph/0601175].

[32] C.S.Li, R.J.Oakes, J.M.Yang and H.Y.Zhou, Phys. Rev. D57, 2009 (1998).

[33] A.Sirlin, Phys. Rev. D 22, 971 (1980); W.J.Marciano and A.Sirlin, ibid. 22, 2695 (1980); A.Sirlin and W.J.Marciano, Nucl. Phys. B189, 442 (1981); K.I.Aoki et al., Prog. Theor. Phys. Suppl. 73, 1 (1982).

[34] J. Collins, F.Wilczek, and A. Zee, Phys.Rev.D18, 242 (1978); W.J.Marciano, Phys. Rev. D29, 580 (1984); P. Nason, S. Dawson, and R.K. Ellis, Nucl. Phys. B327, 49 (1989); Nucl. Phys. B335, 260 (1989) (E)

[35] S. G. Gorishny et al., Mod. Phys. Lett. A 5, 2703(1990); Phys. Rev. D 43, 1633(1991); A. Djouadi et al., Z. Phys. C 70 427(1996); Comput. Phys. Commun. 108, 56(1998); M. Spira, Fortschr. Phys. 46, 203(1998).

[36] Review of Particle Physics(2004).

[37] J.Pumplin et al., J.High Energy Phys. 07(2002) 012.

[38] M.Drees and S.P.Martin, hep-ph/9504324.

[39] A.Djouadi et al., hep-ph/0211331.

[40] V. M. Abazov et al., Fermilab-Pub-06/06-077-E, hep-ex/0604029.

[41] A. Abulencia et al., hep-ex/0512072.

[42] A. Denner, Fortschr. Phys.41, 307 (1993). 

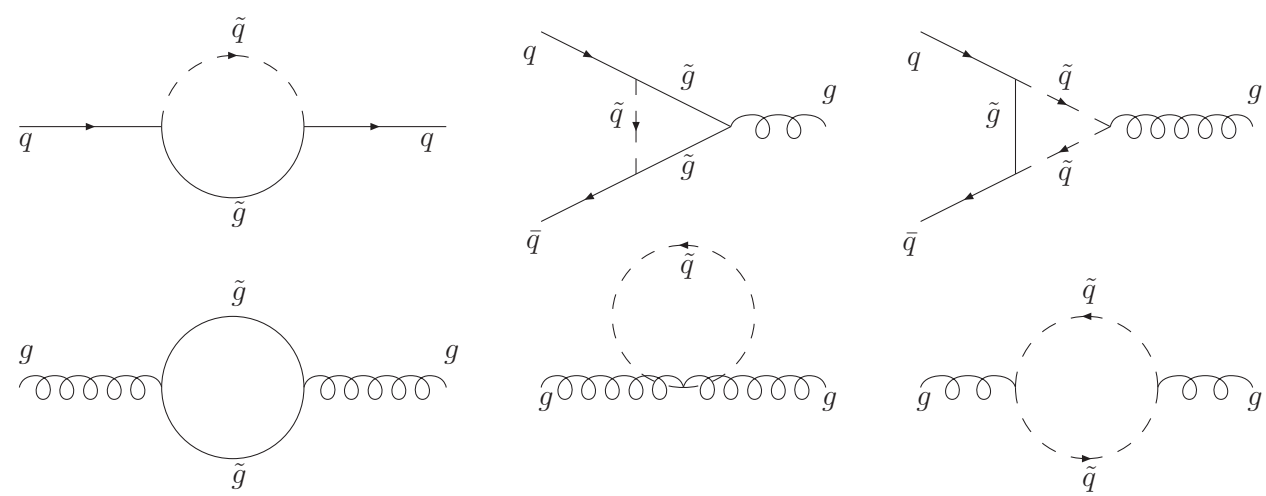

FIG. 1: The self-energy and vertex diagrams for calculating the renormalization constants.
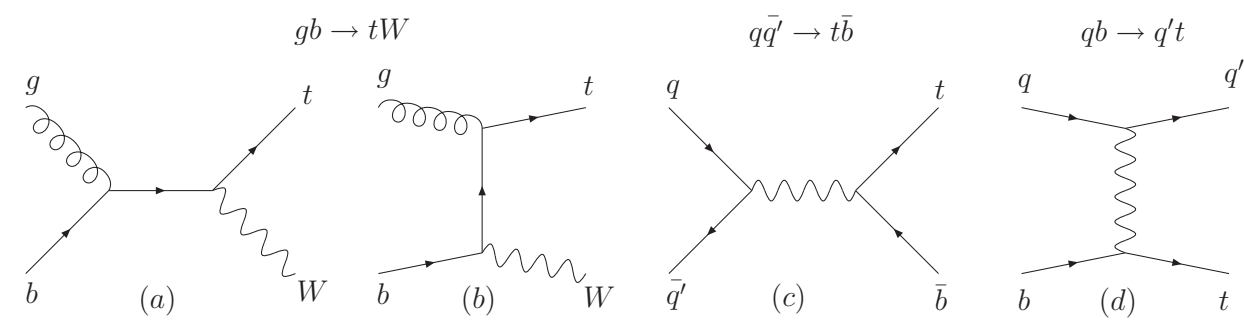

FIG. 2: Tree level Feynman diagrams for associated production.

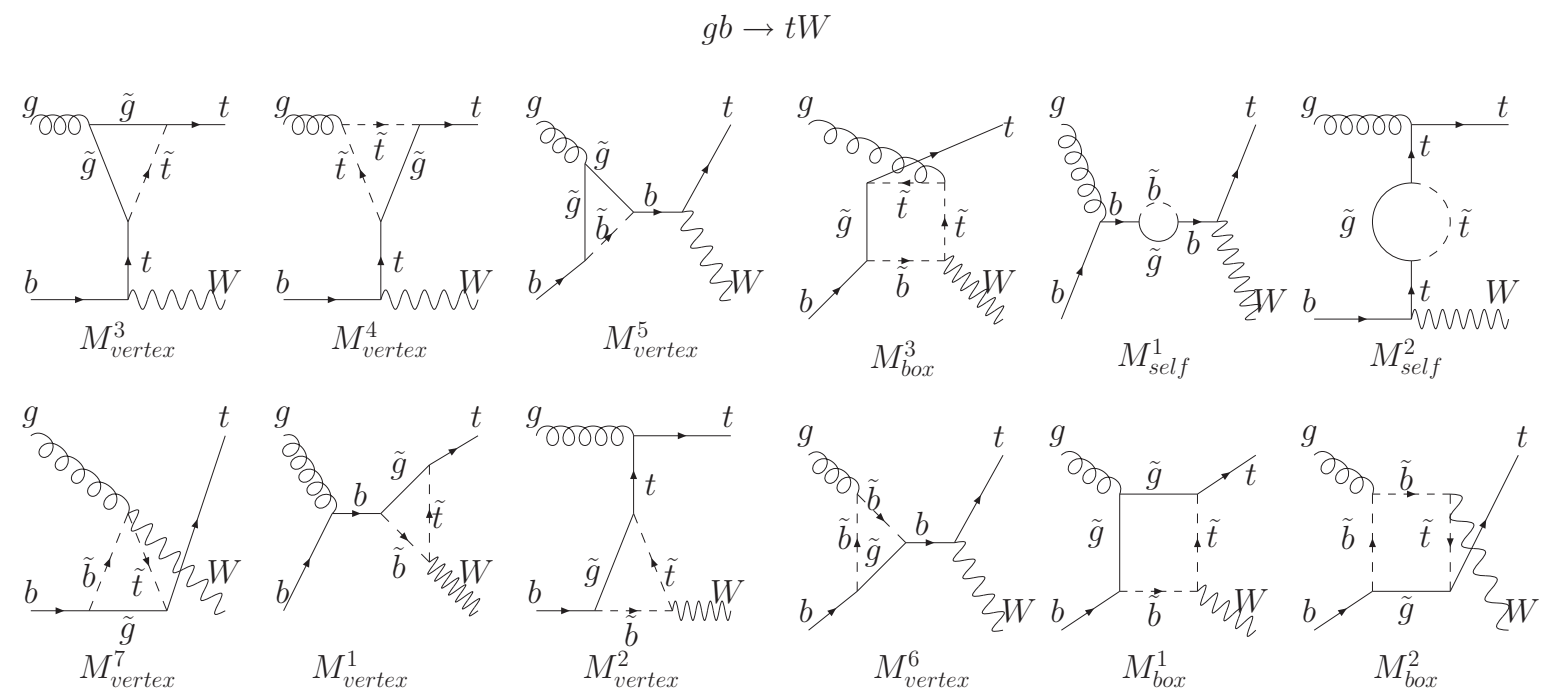

FIG. 3: The Feynman diagrams for SUSY QCD corrections of associate production. 


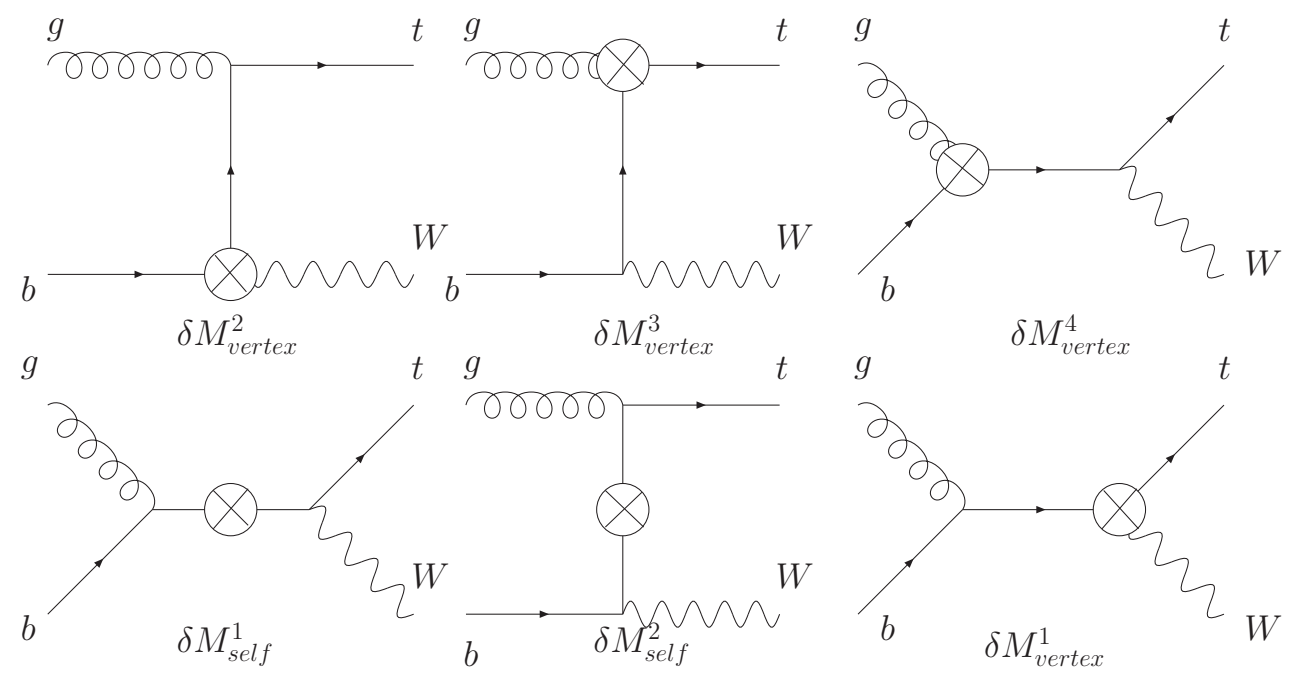

FIG. 4: The counter term diagrams for associated production.

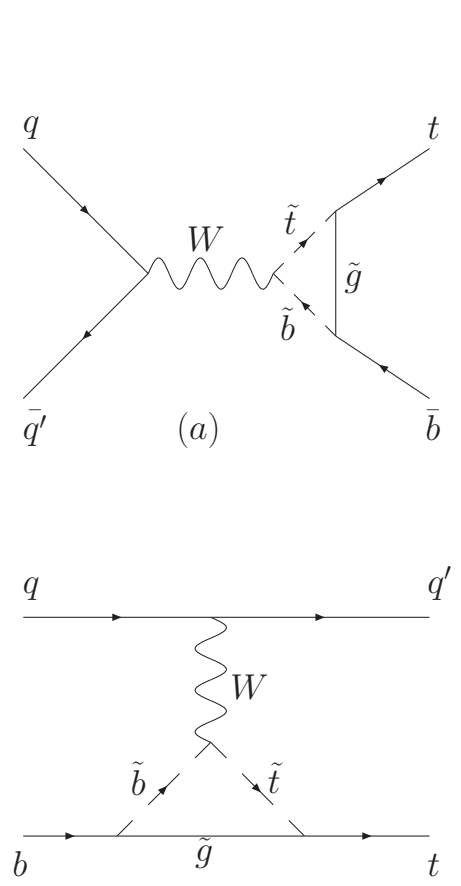

(c)

$q \overline{q^{\prime}} \rightarrow t \bar{b}$

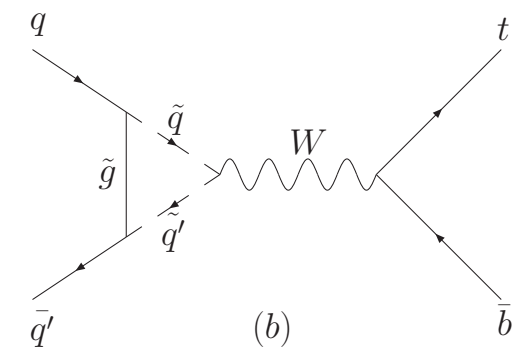

$q b \rightarrow q^{\prime} t$

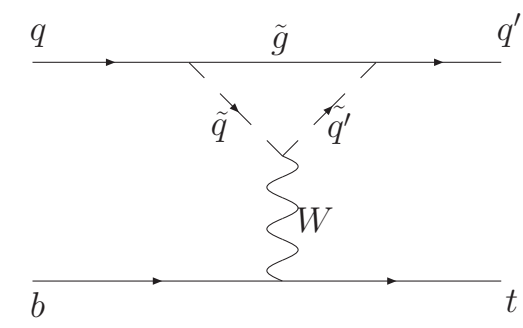

$(d)$

FIG. 5: The Feynman diagrams for s-channel and t-channel loop corrections, respectively. 


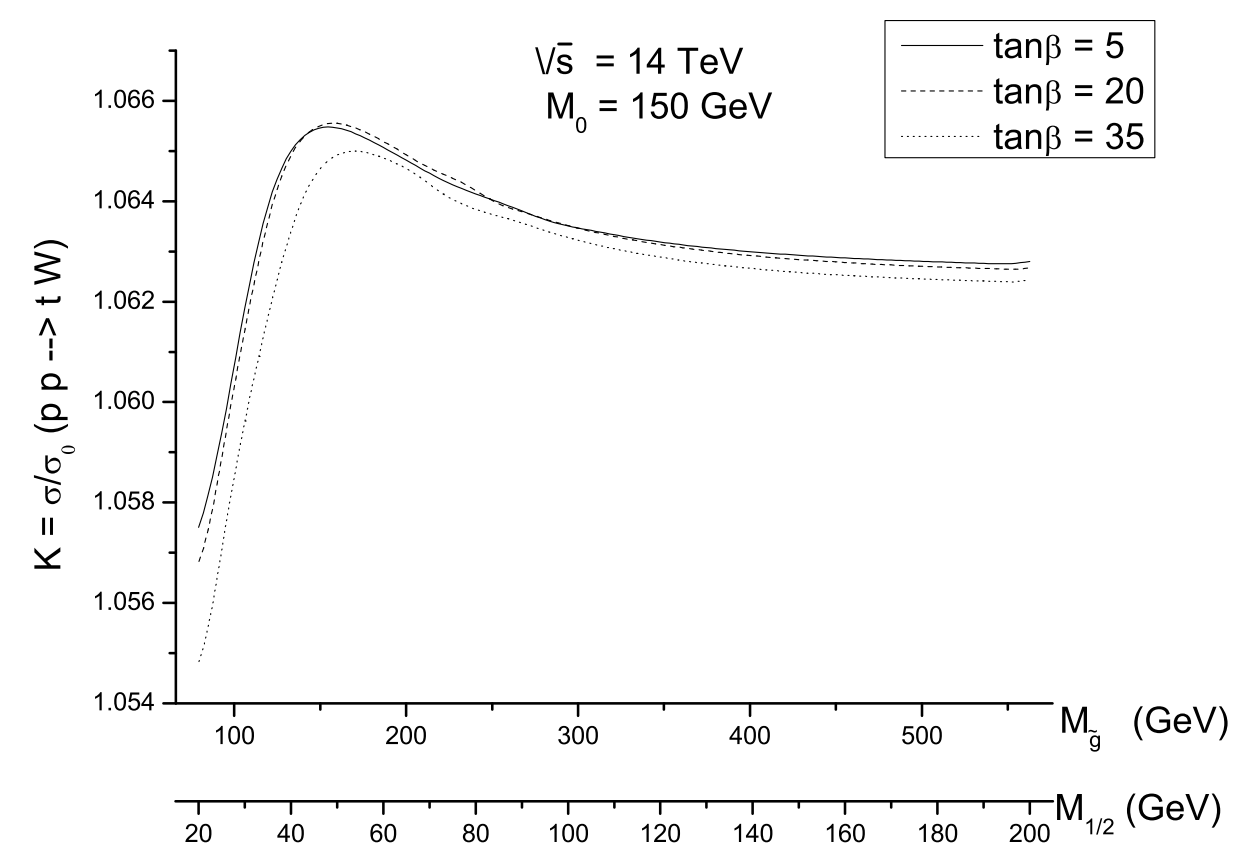

FIG. 6: The K factors as functions of $M_{\tilde{g}}$ or $M_{1 / 2}$ for $p p \rightarrow t W$ at the LHC, where different curves correspond different $\tan \beta$, assuming: $M_{0}=150 \mathrm{GeV}, A_{0}=-200 \mathrm{GeV}$ and $\mu>0$.

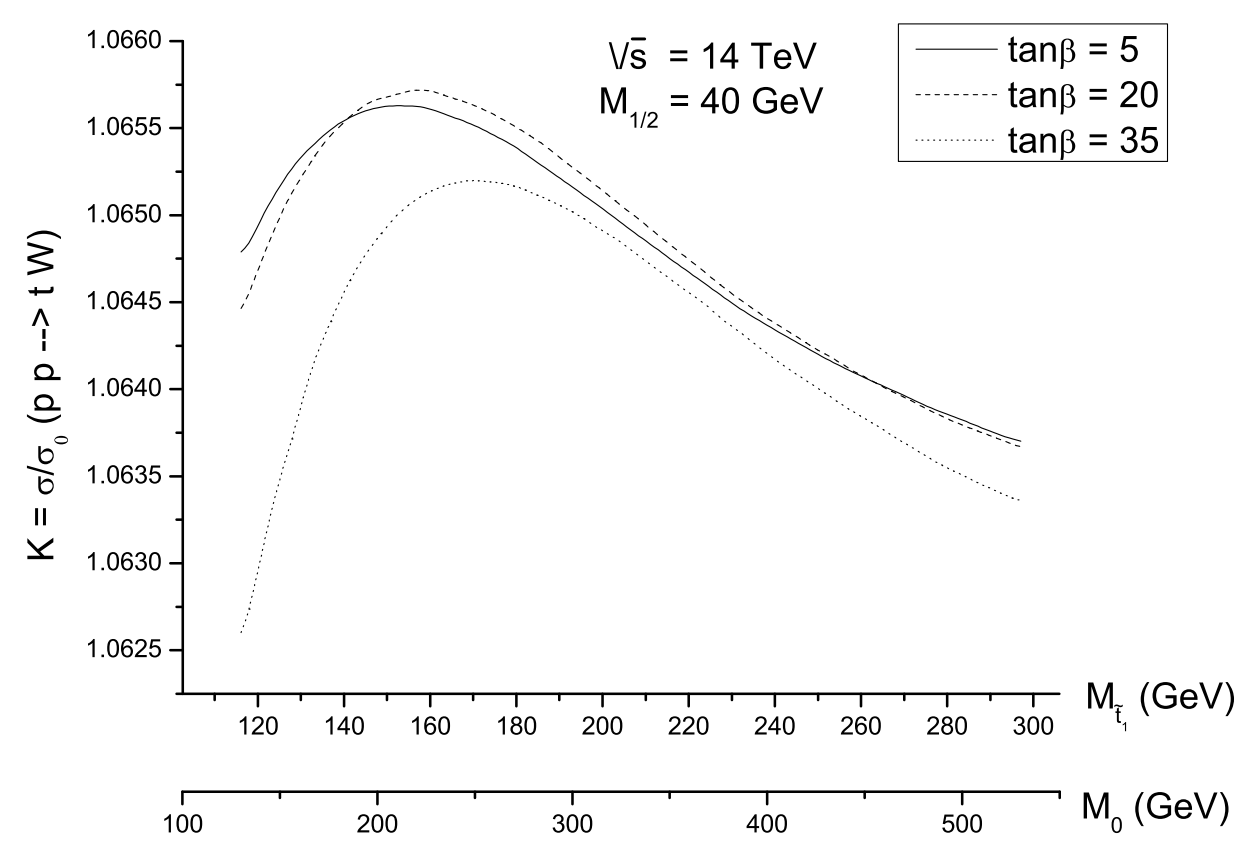

FIG. 7: The $\mathrm{K}$ factors as functions of $M_{\tilde{t}_{1}}$ or $M_{0}$ for $p p \rightarrow t W$ at the LHC, where different curves correspond different tan $\beta$, assuming: $M_{1 / 2}=40 \mathrm{GeV}, A_{0}=-200 \mathrm{GeV}$ and $\mu>0$. 


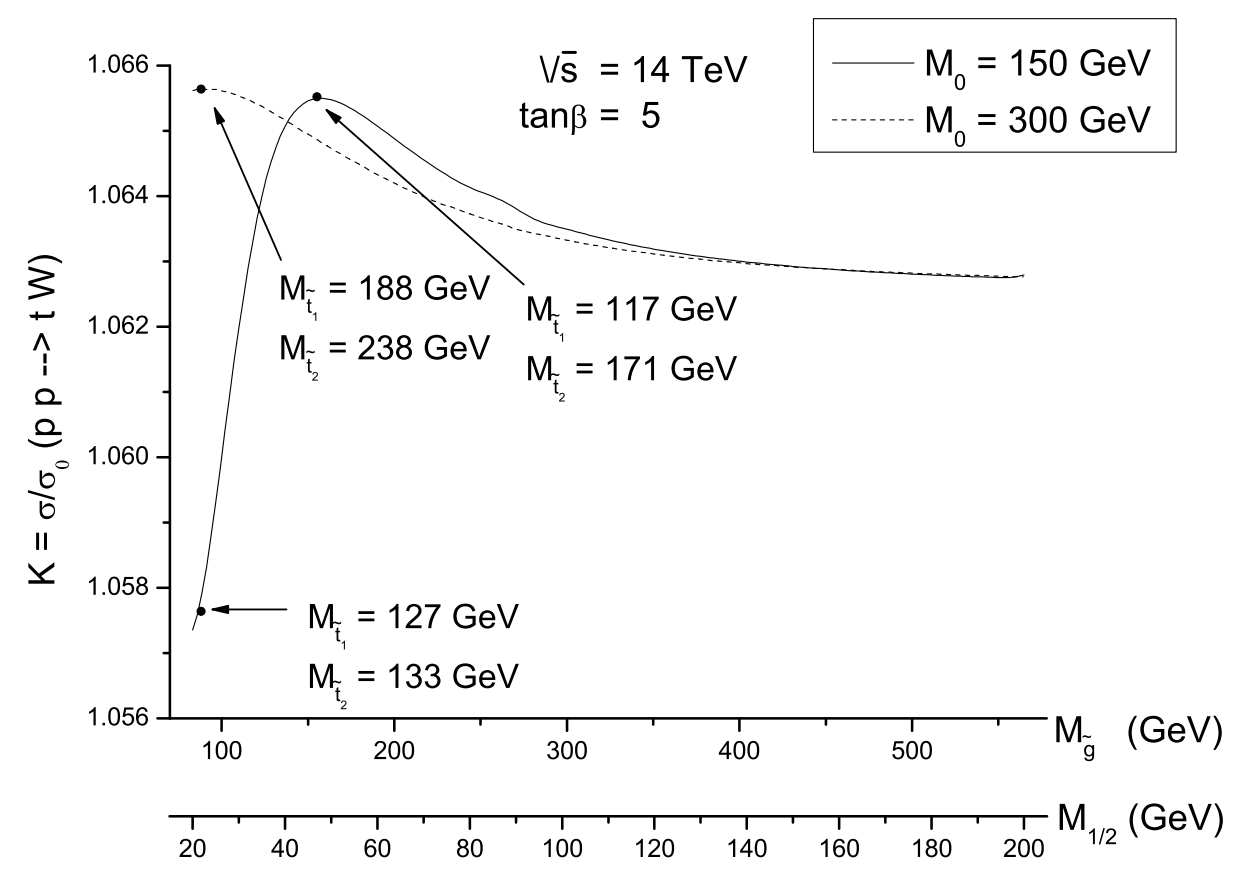

FIG. 8: The K factors as functions of $M_{\tilde{g}}$ or $M_{1 / 2}$ for $p p \rightarrow t W$ at the LHC, where different curves correspond different $M_{0}$, assuming: $\tan \beta=5, A_{0}=-200 \mathrm{GeV}$ and $\mu>0$.

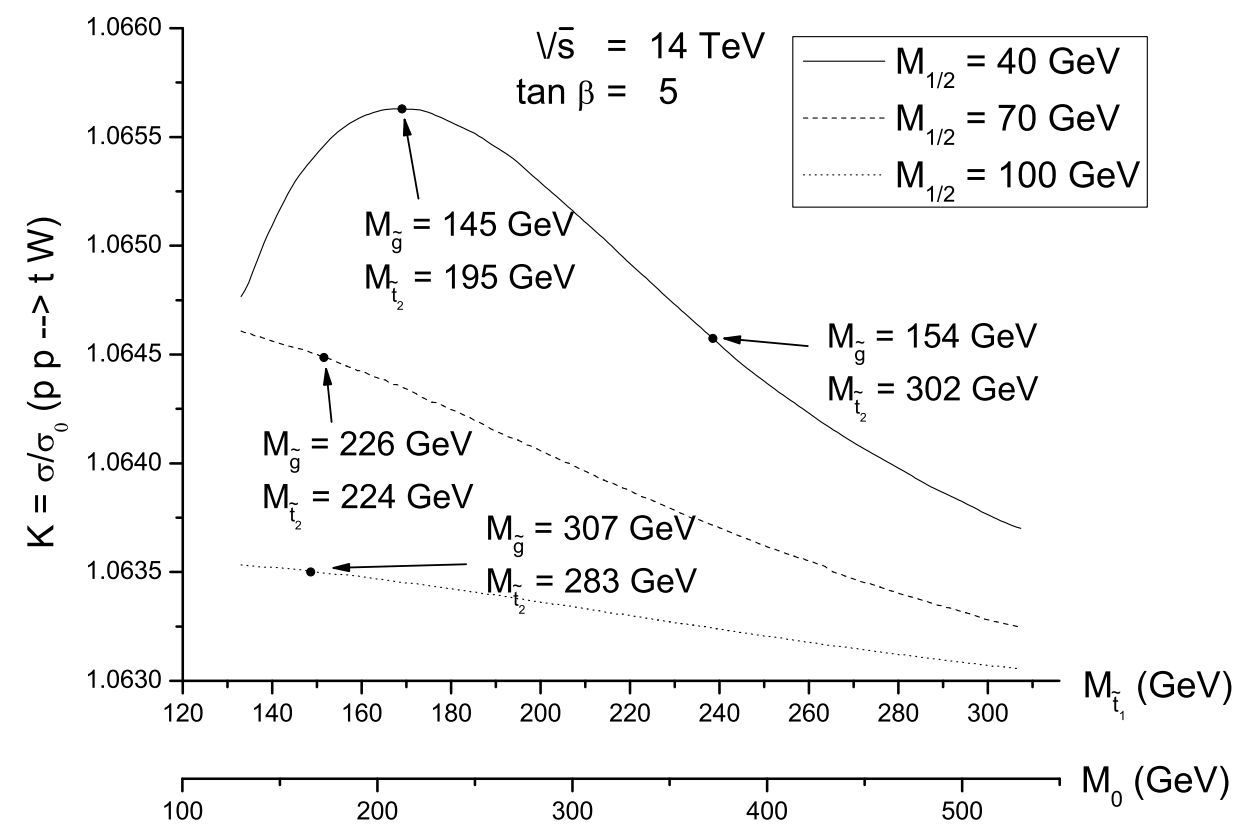

FIG. 9: The K factors as functions of $M_{\tilde{t}_{1}}$ or $M_{0}$ for $p p \rightarrow t W$ at the LHC, where different curves correspond different $M_{1 / 2}$, assuming: $\tan \beta=5, A_{0}=-200 \mathrm{GeV}$ and $\mu>0$. 


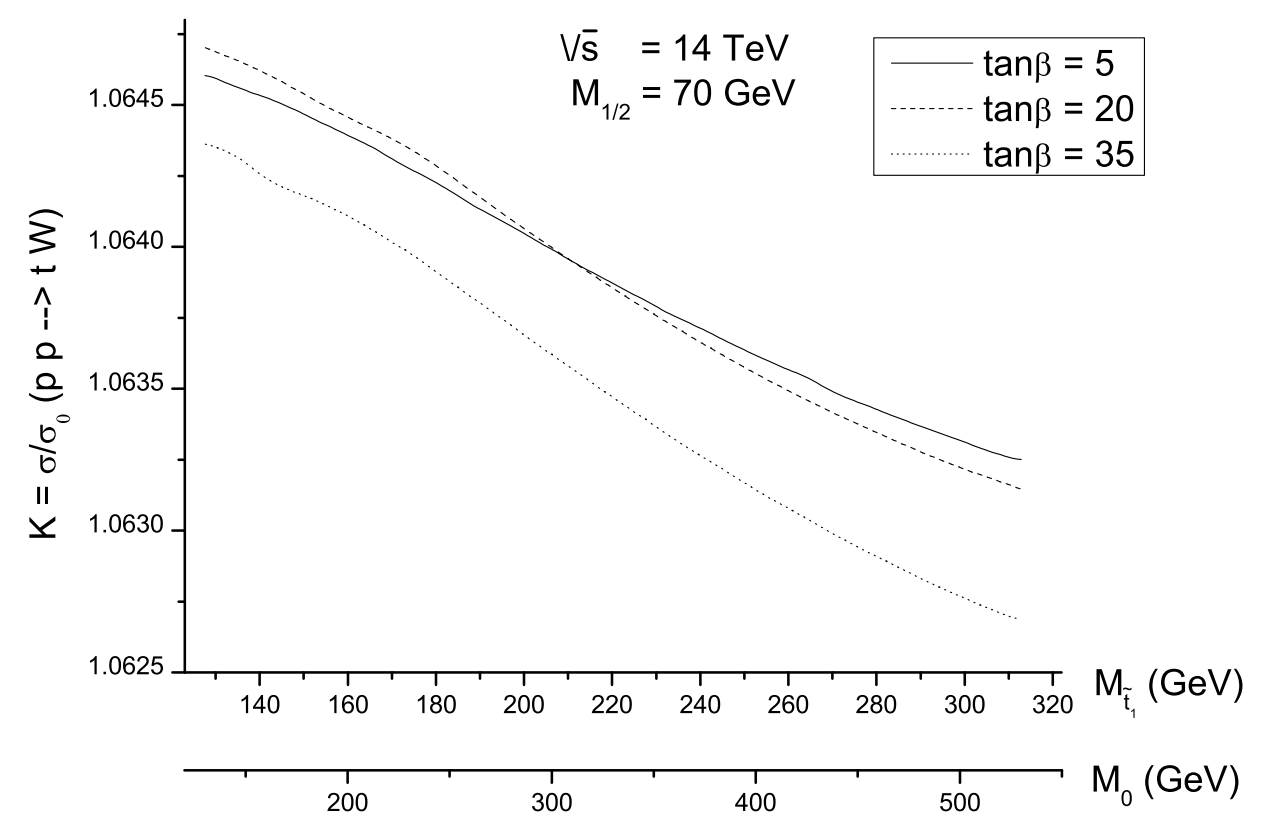

FIG. 10: The K factors as functions of $M_{\tilde{t}_{1}}$ or $M_{0}$ for $p p \rightarrow t W$ at the LHC, where different curves correspond different tan $\beta$, assuming: $M_{1 / 2}=70 \mathrm{GeV}, A_{0}=-200 \mathrm{GeV}$ and $\mu>0$.

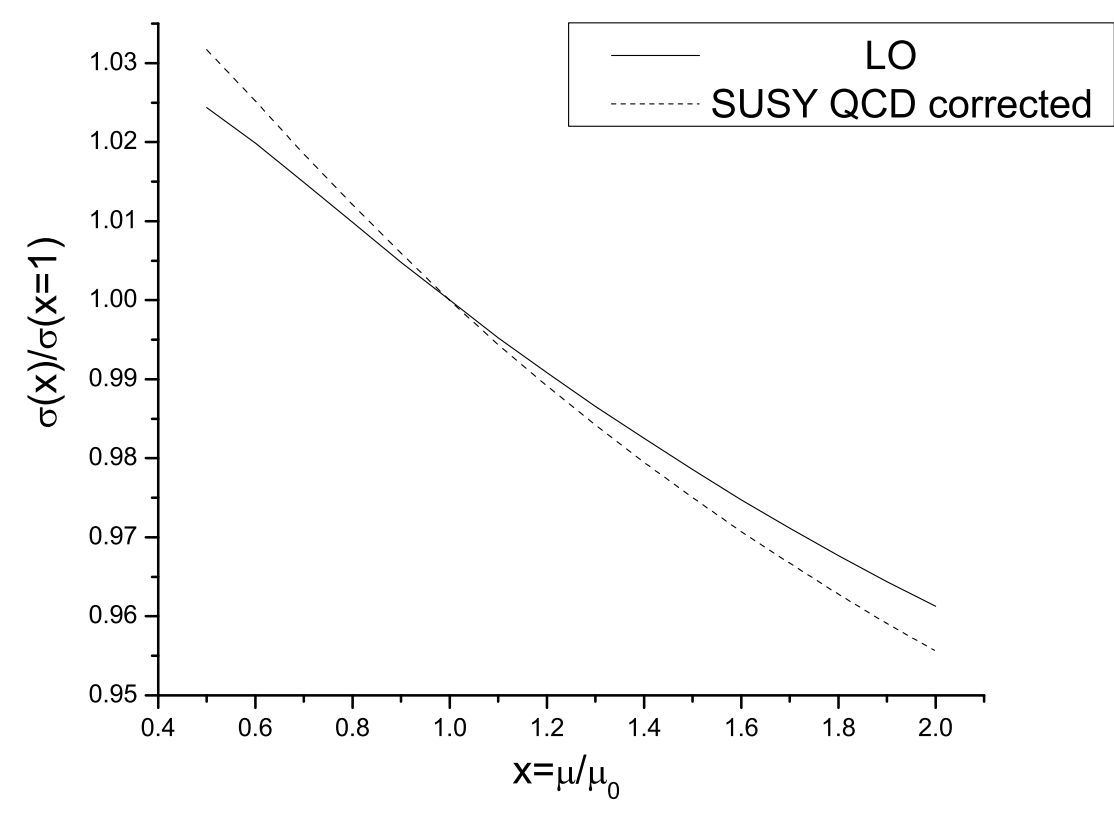

FIG. 11: The scale dependence of LO and SUSY QCD corrected cross sections of associated production at the LHC, we set the factorization and renormalization scales as $\mu_{f}=\mu_{r}=\mu$, and $\mu_{0}=m_{W}+m_{t}$, assuming: $\tan \beta=5, M_{0}=150 \mathrm{GeV}, M_{1 / 2}=70 \mathrm{GeV}, A_{0}=-200 \mathrm{GeV}$ and $\mu>0$. 


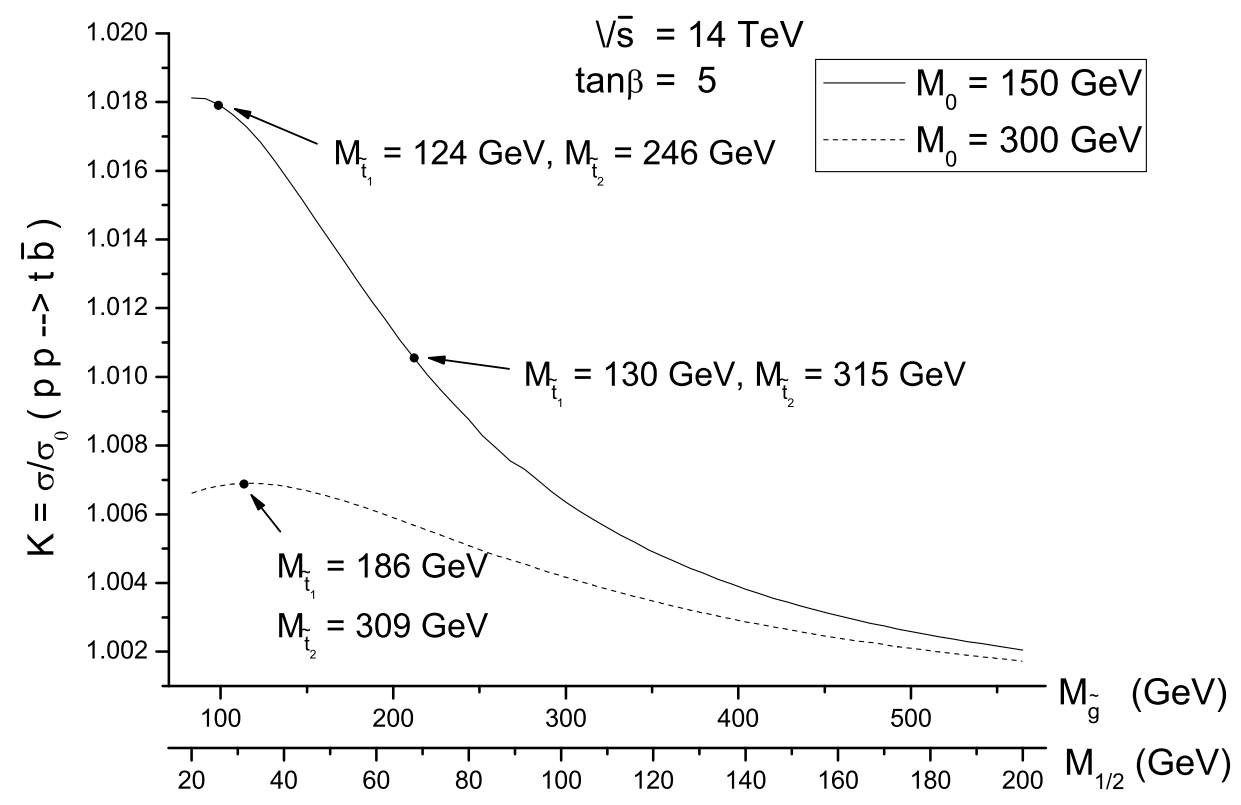

(a)

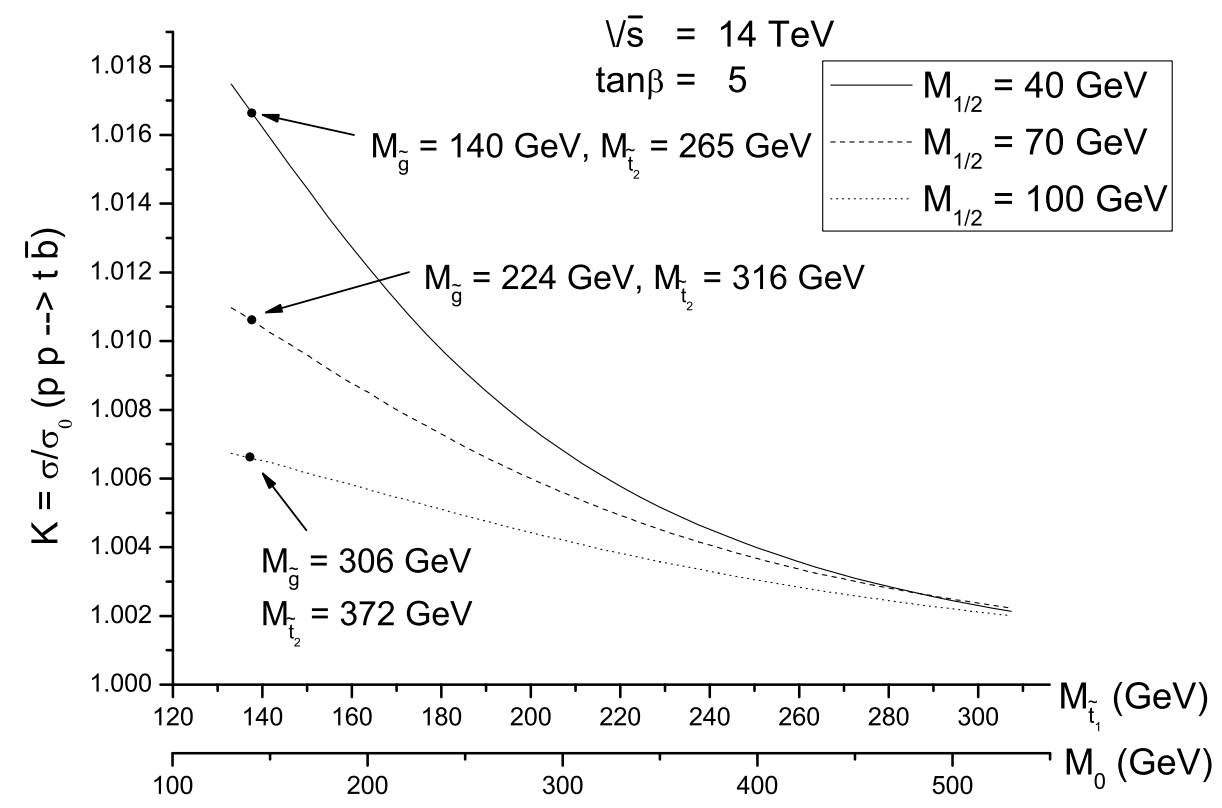

(b)

FIG. 12: The K factors for the $p p \rightarrow t \overline{\mathrm{b}}$ at the LHC. The variables are (a): $M_{\tilde{g}}\left(M_{1 / 2}\right)$ and (b): $M_{\tilde{t}_{1}}\left(M_{0}\right)$, respectively, assuming: $\tan \beta=5, A_{0}=-200 \mathrm{GeV}$ and $\mu>0$. 


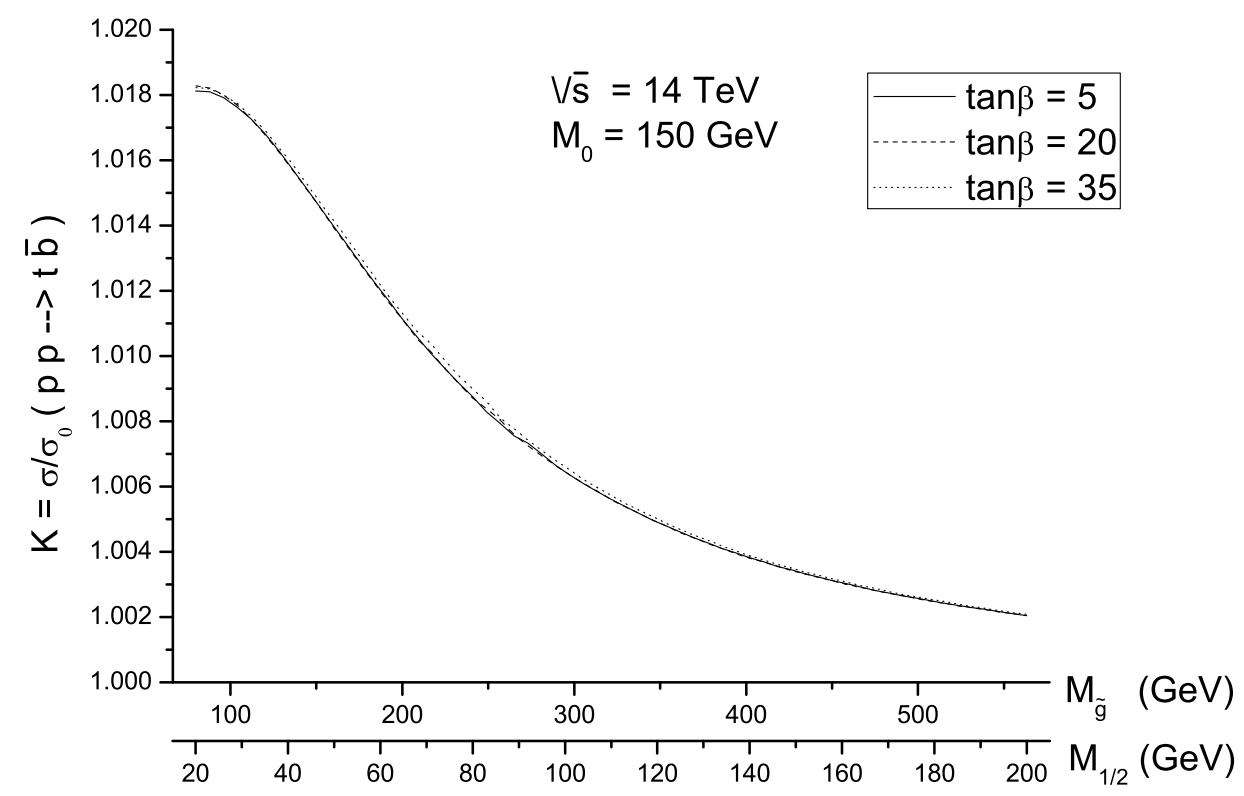

(a)

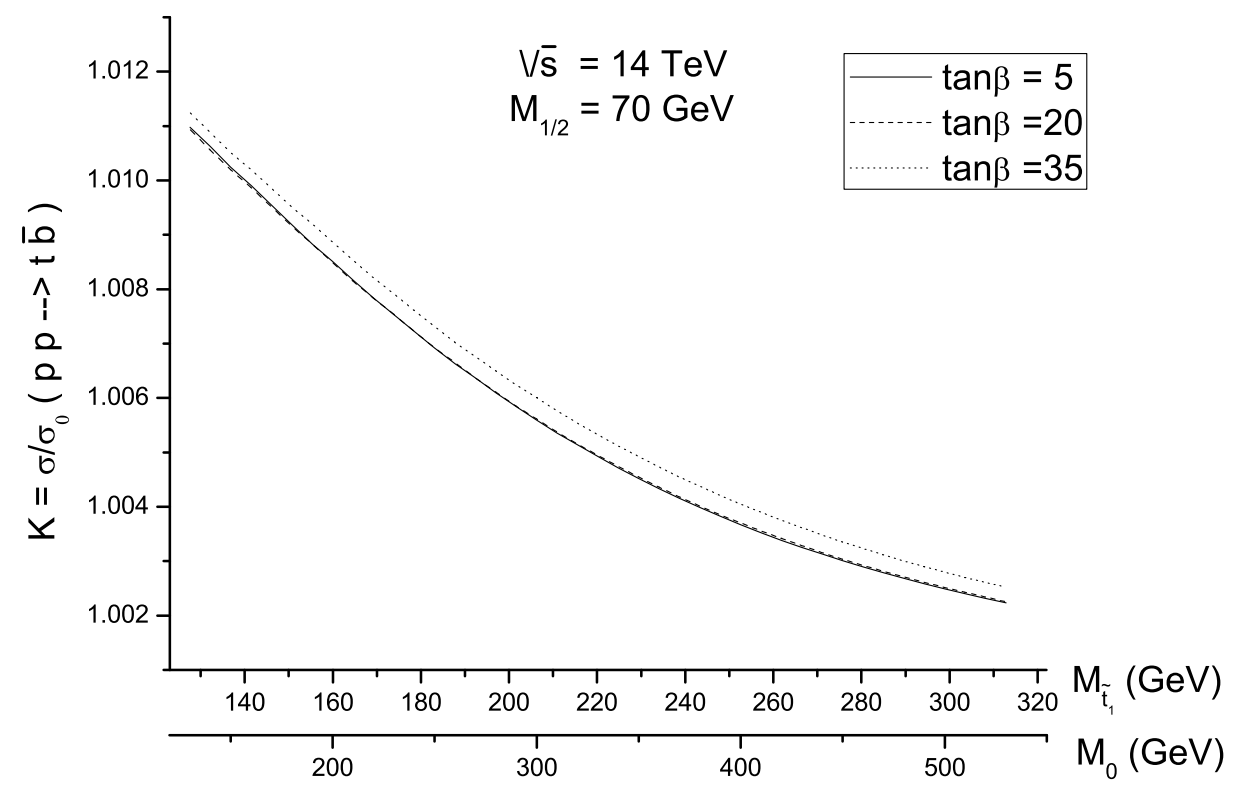

(b)

FIG. 13: The K factors for the $p p \rightarrow t \overline{\mathrm{b}}$ at the LHC. The variables are (a): $M_{\tilde{g}}\left(M_{1 / 2}\right)$ and (b): $M_{\tilde{t}_{1}}\left(M_{0}\right)$, respectively, assuming: $A_{0}=-200 \mathrm{GeV}$ and $\mu>0$. 


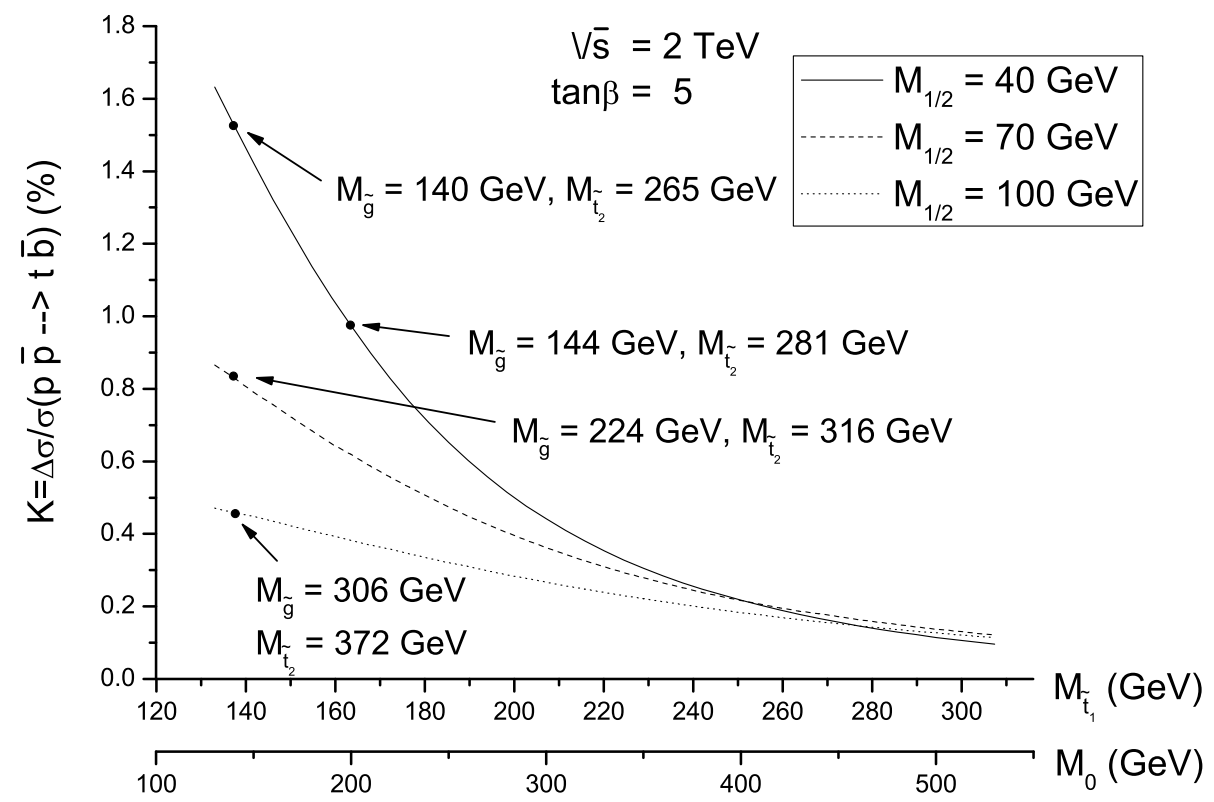

FIG. 14: The $\mathrm{K}$ factors as functions of $M_{\tilde{t}_{1}}\left(M_{0}\right)$ for the $p p \rightarrow t \bar{b}$ at the Tevatron. The graph shows different $M_{1 / 2}$, assuming: $\tan \beta=5, A_{0}=-200 \mathrm{GeV}$ and $\mu>0$.

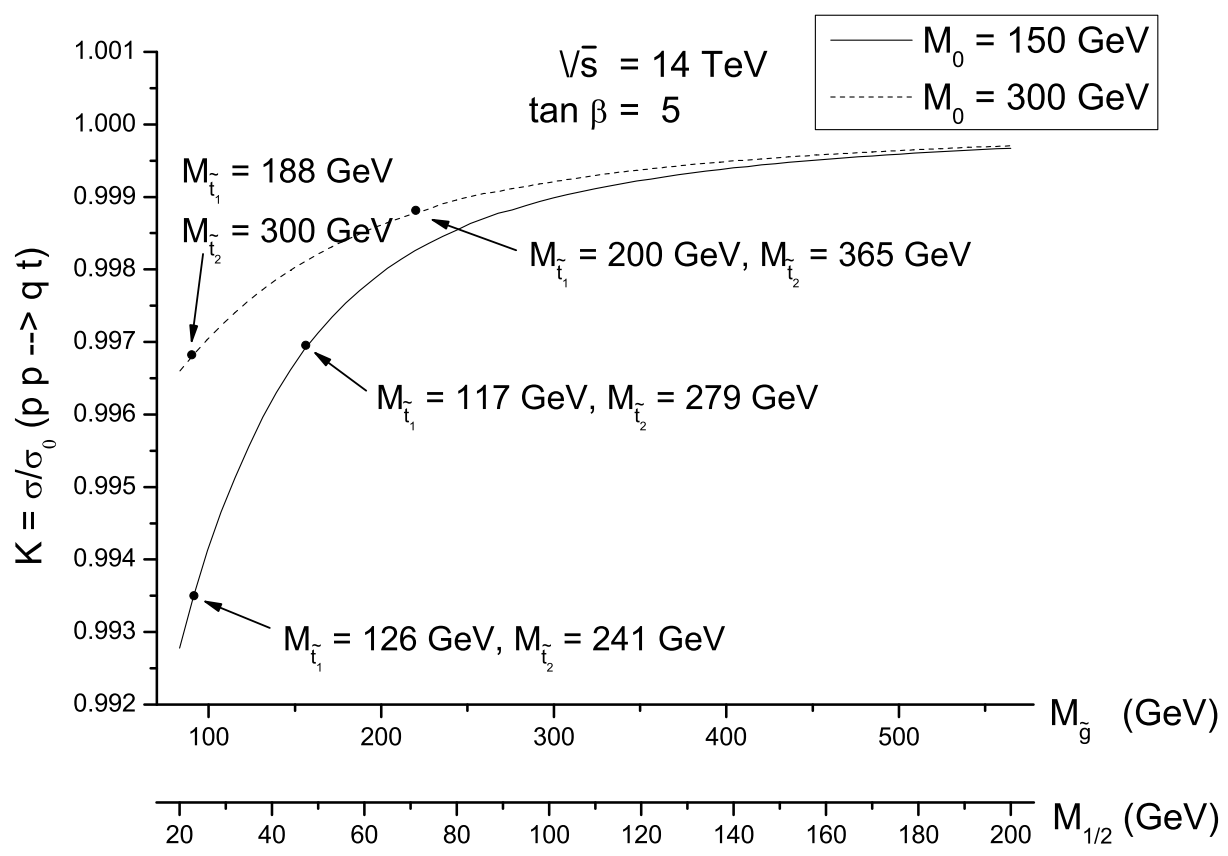

FIG. 15: The K factors as functions of $M_{\tilde{g}}\left(M_{1 / 2}\right)$ for the $p p \rightarrow q t$ at the LHC, the graph shows different $M_{0}$, assuming: $\tan \beta=5, A_{0}=-200 \mathrm{GeV}$ and $\mu>0$. 


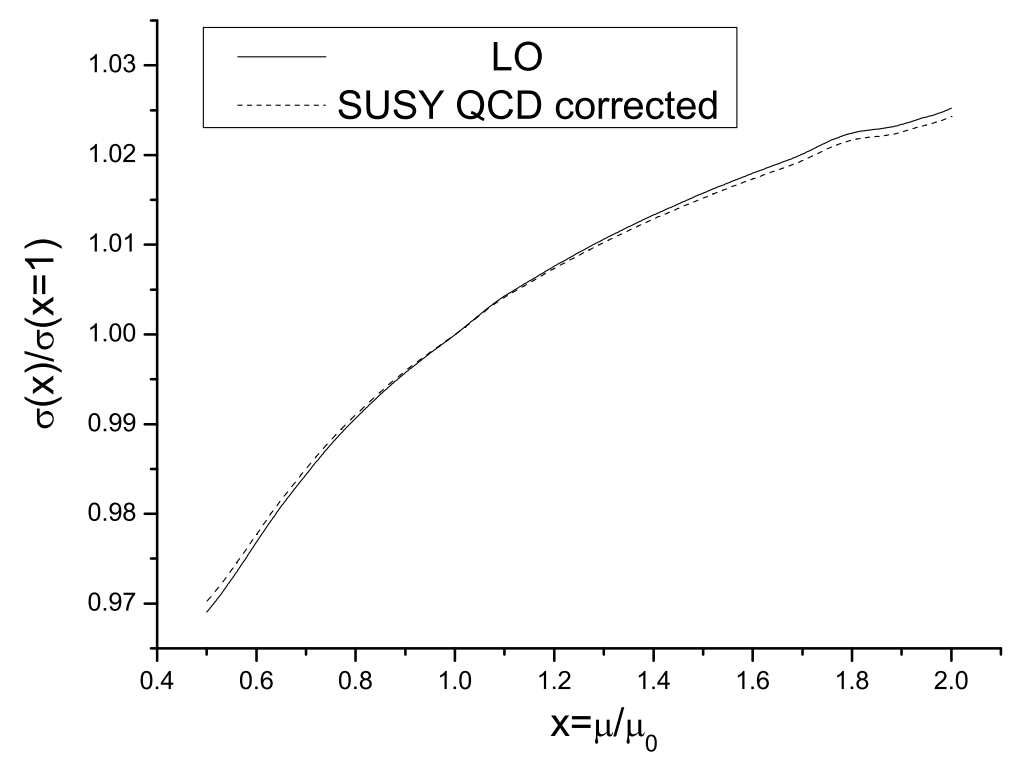

FIG. 16: The scale dependence of LO and SUSY QCD corrected cross sections of s-channel at the LHC, we set the factorization and renormalization scales as $\mu_{f}=\mu_{r}=\mu$, and $\mu_{0}=m_{t}$, assuming: $\tan \beta=5, M_{0}=150 \mathrm{GeV}, M_{1 / 2}=70 \mathrm{GeV}, A_{0}=-200 \mathrm{GeV}$ and $\mu>0$.

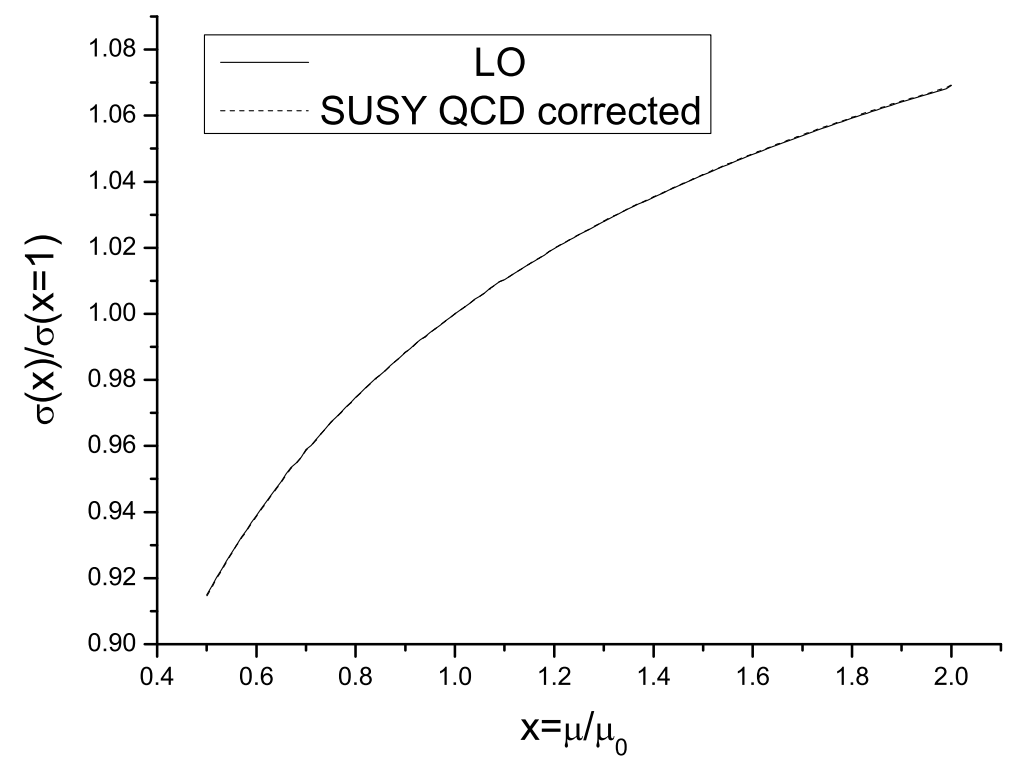

FIG. 17: The scale dependence of LO and SUSY QCD corrected cross sections of t-channel at the LHC, we set the factorization and renormalization scales as $\mu_{f}=\mu_{r}=\mu$, and $\mu_{0}=m_{t}$, assuming: $\tan \beta=5, M_{0}=150 \mathrm{GeV}, M_{1 / 2}=70 \mathrm{GeV}, A_{0}=-200 \mathrm{GeV}$ and $\mu>0$. 